Pacific

Journal of

Mathematics

SINGULARITIES OF FLAT FRONTS IN HYPERBOLIC SPACE

Masatoshi KoKubu, Wayne Rossman, Kentaro Saji, MASAAKI UMEHARA AND KoTARO YAMADA 


\title{
SINGULARITIES OF FLAT FRONTS IN HYPERBOLIC SPACE
}

\author{
Masatoshi Kokubu, Wayne Rossman, Kentaro Saji, \\ MASAAKI UMEHARA AND KOTARO YAMADA
}

\begin{abstract}
It is well-known that the unit cotangent bundle of any Riemannian manifold has a canonical contact structure. A surface in a Riemannian 3-manifold is called a front if it is the projection of a Legendrian immersion into the unit cotangent bundle. We give easily computable criteria for a singular point on a front to be a cuspidal edge or a swallowtail. Using this, we prove that generically flat fronts in hyperbolic 3-space admit only cuspidal edges and swallowtails. We also show that any complete flat front (provided it is not rotationally symmetric) has associated parallel surfaces whose singularities consist of only cuspidal edges and swallowtails.
\end{abstract}

\section{Introduction}

It is well-known that the unit cotangent bundle of any Riemannian $n$-manifold $N^{n}$ has a canonical contact structure. Let $M^{2}$ be a 2-manifold and $f: M^{2} \rightarrow N^{3}$ a $C^{\infty}$ map. We call $f$ a wave front, or front for short, if it is the projection of a Legendrian immersion into the unit cotangent bundle of $N^{3}$. Now let $f: M^{2} \rightarrow \widetilde{M}^{3}(c)$ be a front, where $\tilde{M}^{3}(c)$ is the space form of constant curvature $c$. The associated parallel front $f_{t}: M^{2} \rightarrow \widetilde{M}^{3}(c)$, i.e. the surface that is equidistant from $f$ at a distance $t$ (called a parallel surface of $f=f_{0}$ ), is well-defined. Moreover, if $f$ is a flat immersion, so is $f_{t}$ for $t$ close to zero. Using this fact, we shall define a flat front: A front $f$ is flat in a neighborhood of $p \in M^{2}$ if either

(1) $p$ is a regular point of $f$ and the Gaussian curvature of $f$ near $p$ vanishes, or

(2) $p$ is a singular point of $f$ and $f_{t}$ is a flat immersion around $p$ for all $t \neq 0$ close to zero.

A front $f: M^{2} \rightarrow \tilde{M}^{3}(c)$ is called a flat front if it is flat everywhere on $M^{2}$.

For the case $c=0$, several articles have studied the singularities of developable surfaces in $\mathbb{R}^{3}$. In particular, Izumiya and Takeuchi [2003] proved that the set of developable surfaces whose singularities are only cuspidal edges, swallowtails or

MSC2000: primary 53C42; secondary 53A99.

Keywords: flat front, hyperbolic 3-space, cuspidal edge singularity, swallowtail singularity.

Saji was supported by JSPS Research Fellowships for Young Scientists. 
cuspidal cross caps are open and dense in the set of noncylindrical developable surfaces, where $(u, v) \mapsto\left(u, v^{2}, v^{3}\right)$ represents a cuspidal edge, $(u, v) \mapsto\left(3 u^{4}+\right.$ $\left.u^{2} v, 4 u^{3}+2 u v, v\right)$ a swallowtail, and $(u, v) \mapsto\left(u, u v^{3}, v^{2}\right)$ a cuspidal cross cap. Recently, geometric inequalities for complete flat fronts in hyperbolic 3-space and complete maximal surfaces with certain singularities in Minkowski 3-space were found in [Kokubu et al. 2004] and [Fujimori et al. 2005]. Kitagawa [1988; 1995; 2000] has made a deep investigation of flat tori in the 3-sphere. The study of global properties of surfaces with singularities is a newly developing research area in differential geometry.

This paper investigates singularities of flat surfaces in hyperbolic 3-space $\mathrm{H}^{3}=$ $\widetilde{M}^{3}(-1)$. The geometry of flat fronts in $H^{3}$ has been studied in [Kokubu et al. 2003; 2004]; the latter article gives an analogue of the Osserman inequality for minimal surfaces in $\mathbb{R}^{3}$. Like constant mean curvature one surfaces in $H^{3}$, flat surfaces have a representation formula in terms of holomorphic data, found by J. A. Gálvez, A. Martínez and F. Milán [Gálvez et al. 2000]: Let $\omega$ and $\theta$ be holomorphic 1-forms on a simply-connected Riemann surface $M^{2}$ such that $|\omega|^{2}+|\theta|^{2}$ is positive definite. Then there exists a holomorphic immersion $E_{f}: M^{2} \rightarrow \operatorname{SL}(2, \mathbb{C})$ such that

$$
E_{f}^{-1} d E_{f}=\left(\begin{array}{cc}
0 & \theta \\
\omega & 0
\end{array}\right)
$$

and its projection to $H^{3}$ gives a flat front $f=E_{f} E_{f}^{*}$ in $H^{3}$, where we regard $H^{3}$ as

$$
H^{3}=\mathrm{SL}(2, \mathbb{C}) / \mathrm{SU}(2)=\left\{a a^{*} ; a \in \operatorname{SL}(2, \mathbb{C})\right\} \quad\left(a^{*}={ }^{t} \bar{a}\right) .
$$

Moreover, any simply-connected flat front has such a representation with respect to the complex structure induced by the second fundamental form; see [Gálvez et al. 2000; Kokubu et al. 2003; 2004]. We call $\omega$ and $\theta$ in (1-1) the canonical forms of $f$. In Section 3 of this paper, we will show:

Theorem 1.1. Let $f: M^{2} \rightarrow H^{3}$ be a flat front with canonical forms $(\omega=\hat{\omega} d z$, $\theta=\hat{\theta} d z$ ), where $z$ is a local complex coordinate.

(1) A point $p \in M^{2}$ is a singular point if and only if $|\hat{\omega}(p)|=|\hat{\theta}(p)|$.

(2) The image of $f$ around a singular point $p$ is locally diffeomorphic to a cuspidal edge if and only if

$$
\hat{\omega}^{\prime} \hat{\theta}-\hat{\theta}^{\prime} \hat{\omega} \neq 0 \quad \text { and } \quad \operatorname{Im} \frac{\left(\hat{\theta}^{\prime} / \hat{\theta}\right)-\left(\hat{\omega}^{\prime} / \hat{\omega}\right)}{\sqrt{\hat{\omega} \hat{\theta}}} \neq 0
$$

at $p$, where' $=d / d z$. 
(3) The image of $f$ around a singular point $p$ is locally diffeomorphic to a swallowtail if and only if

$$
\hat{\omega}^{\prime} \hat{\theta}-\hat{\theta} \hat{\omega}^{\prime} \neq 0, \quad \operatorname{Im} \frac{\left(\hat{\theta}^{\prime} / \hat{\theta}\right)-\left(\hat{\omega}^{\prime} / \hat{\omega}\right)}{\sqrt{\hat{\omega} \hat{\theta}}}=0 \quad \text { and } \quad \operatorname{Re} \frac{s(\hat{\theta})-s(\hat{\omega})}{\hat{\omega} \hat{\theta}} \neq 0
$$

at $p$, where $s(\hat{\omega})$ is the Schwarzian derivative of the function $h(z):=\int_{z_{0}}^{z} \omega$ with respect to $z$ :

$$
s(\hat{\omega})=\{h, z\}=\left(\frac{h^{\prime \prime}}{h^{\prime}}\right)^{\prime}-\frac{1}{2}\left(\frac{h^{\prime \prime}}{h^{\prime}}\right)^{2}=\left(\frac{\hat{\omega}^{\prime}}{\hat{\omega}}\right)^{\prime}-\frac{1}{2}\left(\frac{\hat{\omega}^{\prime}}{\hat{\omega}}\right)^{2} .
$$

Consequently, cuspidal edges and swallowtails are stable under perturbations of $(\omega, \theta)$. It is well-known that generic fronts (which might not be flat) admit only cuspidal edges or swallowtails; [Arnol'd et al. 1985, Section 21.6]. However, it does not immediately follow that the set of such fronts is dense within the set of flat fronts. Using Theorem 1.1, we shall prove a similar assertion for flat fronts in $H^{3}$ (Theorem 3.4).

A front $f: M^{2} \rightarrow H^{3}$ is called complete if there exist a compact set $C \subset M^{2}$ and a symmetric 2-tensor $T$ on $M^{2}$ such that $T$ is identically 0 outside $C$ and $d s^{2}+T$ is a complete Riemannian metric of $M^{2}$, where $d s^{2}$ is the first fundamental form of $f$. In Section 4 we prove a global result:

Theorem 1.2. Let $f: M^{2} \rightarrow H^{3}$ be a complete flat front which is not a covering of an hourglass (hourglasses are rotationally symmetric - see Example 6.2), and let $\left\{f_{t}\right\}$ be the family of parallel fronts of $f$. Then, except for only finitely many values of $t$, all the singular points of $f_{t}$ are locally diffeomorphic to cuspidal edges or swallowtails.

The image of the singular points under an hourglass is a single point in $H^{3}$, the so-called "cone-like singularity" (see figure on page 344), and any parallel front of an hourglass has the same singularity. Thus the assumption of Theorem 1.2 is necessary.

Fronts which admit only cuspidal edges and swallowtails are called A-mersions, and their topological properties have been investigated by Langevin, Levitt and Rosenberg [Langevin et al. 1995]. The preceding theorem implies that complete flat fronts in $H^{3}$ are generically included in this category.

The union of singular sets for the entire parallel family of a given flat front is called a caustic. (Roitman [2003] has studied the geometric properties of flat surfaces, motivated by a classical result of L. Bianchi; see Section 5.)

To prove Theorem 1.1, we shall give criteria for a singular point on a front to be a cuspidal edge or a swallowtail, as follows: Let $N^{3}$ be a Riemannian 3-manifold, 
$U$ a domain on $\left(\mathbb{R}^{2} ; u, v\right)$, and let

$$
f=f(u, v): U \rightarrow N^{3}
$$

be a $C^{\infty}$-map with a singular point $p \in U$. Then there exist three functions $a, b, c \in$ $C^{\infty}(U)$ such that

$$
d f\left(\frac{\partial}{\partial u}\right) \wedge d f\left(\frac{\partial}{\partial v}\right)=a(u, v) \frac{\partial}{\partial x} \wedge \frac{\partial}{\partial y}+b(u, v) \frac{\partial}{\partial y} \wedge \frac{\partial}{\partial z}+c(u, v) \frac{\partial}{\partial z} \wedge \frac{\partial}{\partial x},
$$

where $(x, y, z)$ is a local coordinate system of $N^{3}$. The rank of a map defined by

$$
\mathscr{G}:(u, v) \mapsto(a(u, v), b(u, v), c(u, v)) \in \mathbb{R}^{3}
$$

does not depend on the choice of local coordinate $(x, y, z)$ nor on the choice of coordinate $(u, v)$. Now we assume $f$ is a front. A singular point $p \in U$ of $f$ is called nondegenerate if the Jacobian matrix of $\varphi$ is of rank one at $p$. There exists a regular curve near a nondegenerate singular point $p$

$$
\gamma=\gamma(t):(-\varepsilon, \varepsilon) \rightarrow U
$$

(called a singular curve) such that $\gamma(0)=p$, and so that the image of $\gamma$ coincides with the set of singularities of $f$ near $p$. The tangential direction of $\gamma(t)$ is called the singular direction, and a nonzero vector $\eta \in T_{\gamma(t)} U$ such that $d f(\eta)=0$ represents the null direction. For each point $\gamma(t)$, vectors in the null direction $\eta(t)$ are uniquely determined up to nonzero scalar multiplication.

Proposition 1.3. Let $N^{3}$ be a Riemannian 3-manifold and $p=\gamma(0) \in U$ be a nondegenerate singular point of a front $f: U \rightarrow N^{3}$.

(1) The germ of the front $f$ at $p$ is locally diffeomorphic to a cuspidal edge if and only if $\eta(0)$ is not proportional to $\dot{\gamma}(0)$, where $\dot{\gamma}(t)=d \gamma(t) / d t$.

(2) The germ of the front $f$ at $p$ is locally diffeomorphic to a swallowtail if and only if $\eta(0)$ is proportional to $\dot{\gamma}(0)$ and

$$
\left.\frac{d}{d t}\right|_{t=0} \operatorname{det}(\dot{\gamma}(t), \eta(t)) \neq 0
$$

We shall prove this proposition in Section 2. These criteria are useful in other situations. In fact, this proposition is applicable for the study of singularities of maximal surfaces in Minkowski space; see [Fujimori et al. 2005].

\section{Criteria for singular points}

Preliminaries. First, we recall from [Bruce and Giblin 1984] some well-known properties for singular points. Let

$$
\varphi=\varphi(w): I \rightarrow \mathbb{R}
$$


be a $C^{\infty}$-function defined on an open interval $I$ containing the origin such that $\varphi(0)=0$. Then $\varphi$ has an $\mathscr{A}_{k}$-singularity at 0 if

$$
\varphi^{\prime}(0)=\varphi^{\prime \prime}(0)=\cdots=\varphi^{(k)}(0)=0 \quad \text { and } \quad \varphi^{(k+1)}(0) \neq 0,
$$

where $\varphi^{\prime}=d \varphi / d w$ and $\varphi^{(j)}=d^{j} \varphi / d w^{j}$. Here, we shall consider the cases $k=2$ and 3.

Let $\Omega$ be an open subset of $\left(\mathbb{R}^{3} ; x, y, z\right)$ containing the origin $\mathbf{0}$. A map

$$
\Phi: I \times \Omega \rightarrow \mathbb{R}
$$

is called an unfolding of $\varphi$ if

$$
\varphi(w)=\Phi(w, \mathbf{0}) .
$$

Moreover, if $\varphi$ has an $\mathscr{A}_{k}$-singularity at 0 and the matrix

$$
\left(\begin{array}{lllll}
\Phi_{x}(0, \mathbf{0}) & \Phi_{x}^{\prime}(0, \mathbf{0}) & \ldots & \Phi_{x}^{(k-1)}(0, \mathbf{0}) \\
\Phi_{y}(0, \mathbf{0}) & \Phi_{y}^{\prime}(0, \mathbf{0}) & \ldots & \Phi_{y}^{(k-1)}(0, \mathbf{0}) \\
\Phi_{z}(0, \mathbf{0}) & \Phi_{z}^{\prime}(0, \mathbf{0}) & \ldots & \Phi_{z}^{(k-1)}(0, \mathbf{0})
\end{array}\right)
$$

is of rank $k$, then $\Phi$ is called a versal unfolding of $\varphi$, where, for example,

$$
\Phi_{x}^{\prime}=\frac{\partial^{2} \Phi}{\partial x \partial w}, \quad \Phi_{x}^{(j)}=\frac{\partial^{j+1} \Phi}{\partial x \partial^{j} w} .
$$

The set

$$
D_{\Phi}:=\left\{\boldsymbol{x} \in \Omega ; \text { there exists a } w \in I \text { with } \Phi(w, \boldsymbol{x})=\Phi^{\prime}(w, \boldsymbol{x})=0\right\}
$$

is called the discriminant set of $\Phi$.

Fact 2.1 [Bruce and Giblin 1984, Section 6]. Suppose $\varphi: I \rightarrow \mathbb{R}$ has an $\mathscr{A}_{k^{-}}$ singularity $(k=2$ or 3$)$ at 0 and $\Phi: I \times \Omega \rightarrow \mathbb{R}$ a versal unfolding of $\varphi$. Then

(1) $D_{\Phi}$ is locally diffeomorphic to a cuspidal edge at $\mathbf{0}$ if $k=2$.

(2) $D_{\Phi}$ is locally diffeomorphic to a swallowtail at $\mathbf{0}$ if $k=3$.

Nondegenerate singular points. Let $N^{3}$ be a Riemannian 3-manifold and $T_{1}^{*} N^{3}$ the unit cotangent bundle. A $C^{\infty}$-map $f: M^{2} \rightarrow N^{3}$ is called a (wave) front if there exists a Legendrian immersion $L_{f}: M^{2} \rightarrow T_{1}^{*} N^{3}$ such that $f=\pi \circ L_{f}$, where $\pi: T_{1}^{*} N^{3} \rightarrow N^{3}$ is the projection. We call $L_{f}$ the Legendrian lift of $f$.

Lemma 2.2 [Zakalyukin 1983]. Let $U \subset \mathbb{R}^{2}$ be a neighborhood of the origin, and let $f_{j}: U \rightarrow \mathbb{R}^{3}(j=1,2)$ be fronts. Suppose that $(0,0)$ is a singular point of $f_{j}$ and the set of regular points of $f_{j}$ is dense in $U$ for each $j=1,2$. The following two statements are equivalent: 
(i) There exist neighborhoods $V_{1}, V_{2}\left(\subset \mathbb{R}^{2}\right)$ of the origin $(0,0)$ and a local diffeomorphism on $\mathbb{R}^{3}$ which maps the image $f_{1}\left(V_{1}\right)$ to $f_{2}\left(V_{2}\right)$, namely the image of $f_{1}$ is locally diffeomorphic to that of $f_{2}$.

(ii) There exists a local diffeomorphism $h$ on $\mathbb{R}^{2}$ and a local contact diffeomorphism $\Phi$ on $T_{1}^{*} \mathbb{R}^{3}$ which sends fibers to fibers such that $\Phi \circ L_{f_{1}}=L_{f_{2}} \circ$ h, namely the lift $L_{f_{1}}$ is Legendrian equivalent to the lift $L_{f_{2}}$.

The lemma is proved in the appendix. We return to the general setting: Since any contact structure is locally equivalent to the canonical contact structure on $T_{1}^{*} \mathbb{R}^{3}$, we may restrict our attention to fronts in the Euclidean 3-space $\mathbb{R}^{3}$. Let $(U ; u, v)$ be a domain in $\mathbb{R}^{2}$ and $f: U \rightarrow \mathbb{R}^{3}$ a front. Identifying the unit cotangent bundle $T_{1}^{*} \mathbb{R}^{3}$ with the unit tangent bundle $T_{1} \mathbb{R}^{3} \simeq \mathbb{R}^{3} \times S^{2}$, there exists a unit vector field

$$
v: U \rightarrow S^{2} \subset \mathbb{R}^{3}
$$

such that the Legendrian lift $L_{f}$ is expressed as $(f, v)$. Since $L_{f}=(f, v)$ is Legendrian,

$$
\langle d f, v\rangle=0 \quad \text { and } \quad\langle v, v\rangle=1
$$

hold, where $\langle$,$\rangle is the Euclidean inner product of \mathbb{R}^{3}$. We call $v$ the unit normal vector field of the front $f$. Then there exists a $C^{\infty}$-function $\lambda \in C^{\infty}(U)$ such that

$$
\frac{\partial f}{\partial u}(u, v) \times \frac{\partial f}{\partial v}(u, v)=\lambda(u, v) v(u, v),
$$

where $\times$ denotes the cross product of $\mathbb{R}^{3}$. Obviously, $(u, v) \in U$ is a singular point of $f$ if and only if $\lambda(u, v)=0$.

Proposition 2.3. A singular point $p \in U$ of a front $f: U \rightarrow \mathbb{R}^{3}$ is nondegenerate if and only if $d \lambda \neq 0$ at $p$.

Proof. Differentiating (2-1) at $p$, we have $d\left(f_{u} \times f_{v}\right)(p)=d \lambda(p) v(p)$. This implies that the rank of $d\left(f_{u} \times f_{v}\right): U \rightarrow \mathbb{R}^{3}$ at $p$ is at most 1 , and that $d \lambda(p) \neq 0$ is equivalent to $d\left(f_{u} \times f_{v}\right)(p) \neq 0$. Therefore, $d \lambda(p) \neq 0$ if and only if $d\left(f_{u} \times f_{v}\right)$ has rank 1 at $p$, that is, the map $\mathscr{G}$ in the introduction has rank 1 at $p$.

Let $p$ be a nondegenerate singular point of a front $f: U \rightarrow \mathbb{R}^{3}$. Since the set of singular points is the set $\{\lambda=0\}$, Proposition 2.3 implies that the set of singular points is parametrized by a smooth curve

$$
\gamma:(-\varepsilon, \varepsilon) \rightarrow U
$$

in a neighborhood of $p$, so that $\gamma(0)=p$. We call the curve $\gamma(t)$ a singular curve passing through $p$, and the direction $\dot{\gamma}(0)$ the singular direction at the singular point $p$, where the dot denotes the derivative with respect to $t$. Since $p$ is a nondegenerate singular point, so is any point $\gamma(t)$ for sufficiently small $t$. Then 
there exists a unique direction $\eta(t) \in T_{\gamma(t)} U$ up to scalar multiplication such that $d f(\eta(t))=0$ for each $t$. We call $\eta(t)$, which is smooth in $t$, the null direction.

Definition 2.4. Let $p$ be a nondegenerate singular point of a front $f: U \rightarrow \mathbb{R}^{3}$, $\gamma(t)$ the singular curve with $\gamma(0)=p$, and $\eta(t)$ the null direction. Then

(1) $p$ is of type $C$ if $\eta(0)$ is not proportional to $\dot{\gamma}(0)$.

(2) $p$ is of type $S$ if $\eta(0)$ is proportional to $\dot{\gamma}(0)$ and

$$
\left.\frac{d}{d t}\right|_{t=0} \operatorname{det}(\dot{\gamma}(t), \eta(t)) \neq 0,
$$

where $\dot{\gamma}(t)$ and $\eta(t)$ are considered as column vectors in $\mathbb{R}^{2}$.

This definition does not depend on the choices of $\gamma$ and $\eta$.

Example 2.5. The map

$$
\hat{f}_{\mathrm{C}}(z, w):=\left(2 w^{3},-3 w^{2}, z\right)
$$

gives a cuspidal edge along the $z$-axis. The null direction is perpendicular to the $z$-axis, and it has a type $\mathrm{C}$ singularity at $(0,0)$.

The map

$$
\hat{f}_{\mathrm{S}}(z, w):=\left(3 w^{4}+z w^{2}, 4 w^{3}+2 w z, z\right)
$$

gives a swallowtail at $(0,0)$. The singular curve is $6 w^{2}+z=0$, and the null direction is parallel to the $w$-axis. So $(0,0)$ is a singularity of type $\mathrm{S}$.

For $\hat{f}=\hat{f}_{\mathrm{C}}$ or $\hat{f}_{\mathrm{S}}$, with components $\hat{f}=\left(\hat{f}_{1}, \hat{f}_{2}, \hat{f}_{3}\right)$, the derivative $\hat{f}_{w}$ vanishes identically on the singular curve; moreover $\hat{f}_{z}(0,0)=(0,0,1)$ and $\hat{f}_{3}(z, w)=z$. We shall now prove that any front $f(u, v)$ can be given such a parametrization $(z, w)$ near a nondegenerate singular point, as follows: We assume that the origin $(0,0)$ of the $u v$-plane is an arbitrarily given nondegenerate singular point of $f$, namely

$$
\lambda(0,0)=0 \quad \text { and } \quad d \lambda(0,0) \neq 0,
$$

and set

$$
f(0,0)=\mathbf{0} .
$$

Proposition 2.6. Suppose that $(0,0)$ is a nondegenerate singular point of a front $f: U \rightarrow \mathbb{R}^{3}$. Then there is a diffeomorphism

$$
\Psi:(V ; z, w) \mapsto(U ; u, v)
$$

with $\Psi(0,0)=(0,0)$ and a rotation at the origin

$$
\Theta: \mathbb{R}^{3} \rightarrow \mathbb{R}^{3}
$$


such that

$$
\hat{f}(z, w)=\left(\hat{f}_{1}(z, w), \hat{f}_{2}(z, w), \hat{f}_{3}(z, w)\right)=\Theta \circ f \circ \Psi(z, w): V \rightarrow \mathbb{R}^{3}
$$

satisfies the following properties:

(1) $\hat{f}_{z}(0,0)=(0,0,1)$.

(2) $\hat{f}_{3}(z, w)=z$.

(3) The derivative $\hat{f}_{w}$ vanishes identically along the singular curve. In particular, $\hat{f}_{w}(0,0)=(0,0,0)$.

(4) If $(0,0)$ is of type $C$, the tangent vector $\partial / \partial z \in T_{(0,0)} V$ can be chosen to be the singular direction at the origin of $V$.

Proof. Let $\gamma(t)$ be the singular curve passing through $(0,0)$. The null direction $\eta(t)$ can be extended to a vector field $\tilde{\eta}$ on $U$, that is,

$$
\eta(t)=\tilde{\eta} \circ \gamma(t) .
$$

On the other hand, we take a vector $\xi_{0} \in T_{(0,0)} U$ which is not proportional to $\eta(0)$ and satisfies

$$
\left|d f\left(\xi_{0}\right)\right|=1 .
$$

If $(0,0)$ is of type $\mathrm{C}$, we choose $\xi_{0}$ to be proportional to $\dot{\gamma}(0)$. Then there exists a vector field $\xi$ on $U$ such that

$$
\xi(0,0)=\xi_{0} .
$$

The vector fields $\xi$ and $\tilde{\eta}$ are linearly independent in a neighborhood of the origin. Hence by a lemma in [Kobayashi and Nomizu 1963, page 182], there exists a new coordinate system $(\tilde{u}, \tilde{v})$ such that $\tilde{u}(0,0)=\tilde{v}(0,0)=0$ and $\partial / \partial \tilde{u}, \partial / \partial \tilde{v}$ are proportional to $\xi, \tilde{\eta}$, respectively. Scaling $\xi$ and $\tilde{\eta}$, we may assume

$$
\frac{\partial}{\partial \tilde{u}}=\xi \quad \text { and } \quad \frac{\partial}{\partial \tilde{v}}=\tilde{\eta},
$$

without loss of generality. From now on, we use the coordinates $(\tilde{u}, \tilde{v})$. However, for notational simplicity, we drop the overhead tildes and write $(\tilde{u}, \tilde{v})$ as just $(u, v)$. So we may assume:

- The derivative $f_{v}$ vanishes identically on the singular curve $\gamma(t)$.

- If $(0,0)$ is of type $\mathrm{C}$, the tangent vector $\partial / \partial u \in T_{(0,0)} U$ points in the singular direction at the origin.

Since $f_{u}(0,0)$ has unit length, we can take a rotation at the origin $\Theta: \mathbb{R}^{3} \rightarrow \mathbb{R}^{3}$ which maps $f_{u}(0,0)$ to $(0,0,1)$, and set

$$
\tilde{f}(u, v)=\left(\tilde{f}_{1}(u, v), \tilde{f}_{2}(u, v), \tilde{f}_{3}(u, v)\right)=\Theta \circ f(u, v) .
$$


Then

$$
\tilde{f}_{u}(0,0)=(0,0,1), \quad \tilde{f}_{v}(0,0)=(0,0,0) .
$$

We set

$$
g(u, v, z):=\tilde{f}_{3}(u, v)-z
$$

Since

$$
g_{u}(0,0,0)=\left(\tilde{f}_{3}\right)_{u}(0,0)=1 \neq 0,
$$

there exists a function $u=u(z, v)$ such that $u(0,0)=0$ and $g(u(z, v), v, z)=0$, namely,

$$
\tilde{f}_{3}(u(z, v), v)=z \text {. }
$$

Then by

$$
u=u(z, w), \quad v=w,
$$

$(z, w)$ gives a new coordinate system. We now set

$$
\hat{f}(z, w):=\tilde{f}(u(z, w), w) .
$$

Then assertion (2) follows immediately. By differentiating (2-2), we have

$$
u_{z}(0,0)\left(\tilde{f}_{3}\right)_{u}(0,0)=1,
$$

and we get

$$
u_{z}(0,0)=1 .
$$

Thus, by differentiating (2-3), we have

$$
\hat{f}_{z}(0,0)=u_{z}(0,0) \tilde{f}_{u}(0,0)=(0,0,1),
$$

which implies (1).

On the other hand,

$$
\hat{f}_{w}(z, w)=(\tilde{f}(u(z, w), w))_{w}=\tilde{f}_{u}(u(z, w), w) u_{w}(z, w)+\tilde{f}_{w}(u(w, z), w) .
$$

Since $\tilde{f}_{v}$ vanishes on $\gamma$, so does $\tilde{f}_{w}(u(z, w), w)$. Thus we have

$$
\hat{f}_{w}(z, w)=\tilde{f}_{u}(u(z, w), w) u_{w}(z, w) \text { on } \gamma .
$$

By differentiating $\tilde{f}_{3}(u(z, w), w)=z$ with respect to $w$, we have

$$
u_{w}(z, w)\left(\tilde{f}_{3}\right)_{u}(u(z, w), w)=0 .
$$

Here, $\left(\tilde{f}_{3}\right)_{u}(u(z, w), w)$ does not vanish near $(0,0)$, since $\left(\tilde{f}_{3}\right)_{u}(0,0)=1$. Then we have

$$
u_{w}(z, w)=0
$$


and thus $\hat{f}_{w}$ vanishes on the singular curve, which proves $(3)$. If $(0,0)$ is of type $\mathrm{C}$, then $(0,0,1)$ is proportional to the singular direction of $\hat{f}$. Since $\hat{f}_{z}(0,0)=$ $(0,0,1)$, we have $(4)$.

Remark 2.7. In this proof,

$$
\hat{v}(z, w):=v(u(z, w), w)
$$

gives the unit normal vector field of the (normalized) front $\hat{f}(z, w)$.

In addition to the case of surfaces, we shall define fronts for plane curves.

Definition 2.8. Let $I \subset \mathbb{R}$ be an interval. A map

$$
\sigma=\sigma(w): I \rightarrow \mathbb{R}^{2}
$$

is called a (planar) front if there exists a map

$$
n=n(w): I \rightarrow S^{1} \subset \mathbb{R}^{2}
$$

such that $n(w)$ is perpendicular to $\sigma(w)$ and $w \mapsto(\sigma(w), n(w))$ is an immersion. A point $w=w_{0}$ with $\sigma^{\prime}\left(w_{0}\right)=0$ is called a singular point of the planar front $\sigma(w)$, where ${ }^{\prime}=d / d w$. At such a point, $n^{\prime}\left(w_{0}\right) \neq 0$ by definition.

A planar front is a projection of a Legendrian immersion in the unit cotangent bundle $T_{1}^{*} \mathbb{R}^{2}$ with respect to the canonical contact structure.

In the cases of $\hat{f}_{\mathrm{C}}$ and $\hat{f}_{\mathrm{S}}$ in Example 2.5, one can easily check that their slices $\sigma^{z}: w \mapsto \hat{f}(z, w)$ perpendicular to the $z$-axis give planar fronts. The tangent line of $\sigma^{z}(w)$ is given by

$$
\Phi(w, x, y, z):=n_{1}(z, w)\left(x-\hat{f}_{1}(z, w)\right)+n_{2}(z, w)\left(y-\hat{f}_{2}(z, w)\right)=0,
$$

where $n(z, w)=\left(n_{1}(z, w), n_{2}(z, w)\right)$ is the unit normal vector of $\sigma^{z}(w)$. Then $\sigma^{z}$ is the envelope of this family of tangent lines, and the discriminant set $D_{\Phi}$ characterizes the image of $\hat{f}_{\mathrm{C}}$ and $\hat{f}_{\mathrm{S}}$. According to this observation, we shall prove that $\hat{f}(z, w)$ as in Proposition 2.6, which has type $\mathrm{C}$ or type $\mathrm{S}$ singularities at $(0,0)$, also satisfies that

(a) the slice perpendicular to the $z$-axis gives a planar front,

(b) the set $D_{\Phi}$ of $\Phi$ given by (2-4) is a discriminant set and is locally diffeomorphic to a cuspidal edge or a swallowtail, by applying Fact 2.1.

Now we shall prove (a) for nondegenerate singular points as follows:

Proposition 2.9. Let $(0,0)$ be a nondegenerate singular point of the front

$$
\hat{f}=\hat{f}(z, w): V \rightarrow \mathbb{R}^{3}
$$


satisfying (2) and (3) in Proposition 2.6. Then there exists an $\varepsilon>0$ such that the map defined by

$$
\sigma^{z}: w \mapsto\left(\hat{f}_{1}(z, w), \hat{f}_{2}(z, w)\right) \quad(|z|<\varepsilon)
$$

is a planar front. Moreover, $w$ is a singular point of $\sigma^{z}$ if $(z, w)$ is a singular point of $\hat{f}$.

To prove this we need the following:

Lemma 2.10. Under the assumptions of Proposition 2.9, the derivative $\hat{v}_{w}(0,0)$ is nonzero and perpendicular to $\boldsymbol{e}_{3}:=(0,0,1)$, where $\hat{v}$ is the unit normal vector field of $\hat{f}$.

Proof. Since $\hat{f}$ is a front, it follows from (3) in Proposition 2.6 that $\hat{v}_{w}(0,0)$ does not vanish. Since $\left\langle\hat{f}_{w}, \hat{v}\right\rangle=0$, we have

$$
0=\left\langle\hat{f}_{w}, \hat{v}\right\rangle_{z}=\left\langle\hat{f}_{w z}, \hat{v}\right\rangle+\left\langle\hat{f}_{w}, \hat{v}_{z}\right\rangle
$$

Since $\hat{f}_{w}(0,0)=0$ by (3) in Proposition 2.6, we have

$$
\left\langle\hat{f}_{w z}(0,0), \hat{v}(0,0)\right\rangle=0 .
$$

Thus,

$$
\begin{aligned}
\left\langle\hat{v}_{w}(0,0), \boldsymbol{e}_{3}\right\rangle & =\left\langle\hat{v}_{w}(0,0), \hat{f}_{z}(0,0)\right\rangle \\
& =\left.\frac{\partial}{\partial w}\right|_{(z, w)=(0,0)}\left\langle\hat{v}, \hat{f}_{z}\right\rangle-\left\langle\hat{v}(0,0), \hat{f}_{w z}(0,0)\right\rangle=0,
\end{aligned}
$$

which is the desired conclusion.

Proof of Proposition 2.9. We fix $z$ and let

$$
\sigma(w):=\left(\hat{f}_{1}(z, w), \hat{f}_{2}(z, w), 0\right) .
$$

Then $\sigma(w)$ is a map into the $x y$-plane. By (2) in Proposition 2.6, we have

$$
\sigma(w)=\hat{f}(z, w)-\left\langle\hat{f}(z, w), \boldsymbol{e}_{3}\right\rangle \boldsymbol{e}_{3}=\hat{f}(z, w)-\hat{f}_{3}(z, w) \boldsymbol{e}_{3}=\hat{f}(z, w)-z \boldsymbol{e}_{3}
$$

and

$$
\frac{d}{d w} \sigma(w)=\hat{f}_{w}(z, w)
$$

This implies that a singular point of $\sigma$ is a singular point of $\hat{f}$.

On the other hand, we set

$$
n(w):=\frac{\hat{v}-\left\langle\hat{v}(z, w), \boldsymbol{e}_{3}\right\rangle \boldsymbol{e}_{3}}{\left(1-\left\langle\hat{v}(z, w), \boldsymbol{e}_{3}\right\rangle^{2}\right)^{1 / 2}} .
$$

Since

$$
\left\langle\hat{v}(0,0), \boldsymbol{e}_{3}\right\rangle=\left\langle\hat{v}(0,0), \hat{f}_{z}(0,0)\right\rangle=0,
$$


$n(w)$ is a well-defined unit vector field near $(0,0)$. Moreover,

$$
\begin{aligned}
\left\langle\sigma_{w}(w), n(w)\right\rangle & =\left\langle\hat{f}_{w}(z, w), n(w)\right\rangle=\frac{\left\langle\hat{v}, \hat{f}_{w}\right\rangle-\left\langle\hat{v}, \boldsymbol{e}_{3}\right\rangle\left\langle\boldsymbol{e}_{3}, \hat{f}_{w}\right\rangle}{\left(1-\left\langle\hat{v}(z, w), \boldsymbol{e}_{3}\right\rangle^{2}\right)^{1 / 2}} \\
& =-\frac{\left\langle\hat{v}, \boldsymbol{e}_{3}\right\rangle}{\left(1-\left\langle\hat{v}(z, w), \boldsymbol{e}_{3}\right\rangle^{2}\right)^{1 / 2}}\left(\hat{f}_{3}\right)_{w}=0,
\end{aligned}
$$

where we used the fact that $\hat{f}_{3}(z, w)=z$. Thus, $n(w)$ is a normal vector of $\sigma(w)$. By Lemma 2.10, we have $\left\langle\hat{v}_{w}(0,0), \boldsymbol{e}_{3}\right\rangle=0$, and

$$
\frac{d}{d w} n(0)=\hat{v}_{w}(0,0) \neq 0 \text {. }
$$

Hence $n^{\prime}(w) \neq 0$ for sufficiently small $(z, w)$, and the map $w \mapsto(\sigma(w), n(w))$ is an immersion.

Proof of the criteria. We now prove Proposition 1.3 from the introduction. As pointed out in the beginning of the previous section, it is sufficient to prove the assertion for fronts in the Euclidean 3-space $\mathbb{R}^{3}$. The idea of the proof is as follows: Let $(0,0)$ be a nondegenerate singular point of a front $f=f(u, v): U \rightarrow \mathbb{R}^{3}$. Then by Proposition 2.6, we have a normalized front $\hat{f}(z, w)$. We set

$$
\sigma(z, w):=\left(\hat{f}_{1}(z, w), \hat{f}_{2}(z, w)\right) .
$$

By Proposition 2.9, there exist positive numbers $\varepsilon_{1}$ and $\varepsilon_{2}$ such that

$$
\left(-\varepsilon_{1}, \varepsilon_{1}\right) \ni w \mapsto \sigma(z, w) \in \mathbb{R}^{2}
$$

gives a planar front for $|z|<\varepsilon_{2}$; that is, there exists a unit normal vector field

$$
n=n(z, w):\left(-\varepsilon_{2}, \varepsilon_{2}\right) \times\left(-\varepsilon_{1}, \varepsilon_{1}\right) \rightarrow \mathbb{R}^{2}
$$

such that $\left\langle\sigma_{w}(z, w), n(z, w)\right\rangle=0$. If we set $n=\left(n_{1}, n_{2}\right)$, the equation

$$
n_{1}(z, w)\left(x-\hat{f}_{1}(z, w)\right)+n_{2}(z, w)\left(y-\hat{f}_{2}(z, w)\right)=0
$$

gives the tangent line of the planar front $w \mapsto \sigma(z, w)$, and the image of the planar front is the envelope of these tangent lines. On the other hand, it is well-known that the envelope generated by a family of lines

$$
\{F(w, x, y)=0 ; w \in \mathbb{R}\}
$$

is given by $\left\{(x, y) ; F(w, x, y)=F_{w}(w, x, y)=0, w \in \mathbb{R}\right\}$. So if we set

$$
\Phi(w, x, y, z):=n_{1}(z, w)\left(x-\hat{f}_{1}(z, w)\right)+n_{2}(z, w)\left(y-\hat{f}_{2}(z, w)\right),
$$


the discriminant set

$$
D_{\Phi}:=\left\{\boldsymbol{x} \in \Omega ; \text { there exists a } w \in \mathbb{R} \text { with } \Phi(w, \boldsymbol{x})=\Phi_{w}(w, \boldsymbol{x})=0\right\}
$$

coincides with the image of the front $\hat{f}$. Now we set

$$
\varphi(w)=\Phi(w, 0,0,0)
$$

Then if $\varphi(w)$ has an $\mathscr{A}_{k}$-singularity $(k=2,3)$ and $\Phi$ is a versal unfolding, we can conclude (b), that is, the image of $\hat{f}$ is locally diffeomorphic to a cuspidal edge or a swallowtail, by Fact 2.1. According to this plan, we shall first prove the criterion for cuspidal edges. First, we prepare three lemmas:

Lemma 2.11. $\sigma(0,0)=\sigma^{\prime}(0,0)=\sigma_{z}(0,0)=(0,0)$ and $n^{\prime}(0,0) \neq(0,0)$ hold, where' denotes the derivative with respect to $w$.

Proof. These are easily computed from Propositions 2.6 and 2.9.

Lemma 2.12. $\Phi_{z}(0,0,0,0)=\Phi_{z}^{\prime}(0,0,0,0)=0$.

Proof. These are computed by differentiating (2-5) and by using Lemma 2.11 .

Lemma 2.13. $\varphi(w)$ has an $\mathscr{A}_{2}$-singularity at $w=0$ if and only if $\sigma^{\prime \prime}(0,0) \neq(0,0)$.

Proof. By differentiating $\left\langle\sigma^{\prime}, n\right\rangle=0$ and using $\sigma^{\prime}(0,0)=(0,0)$, we conclude that $\left\langle\sigma^{\prime \prime}(0,0), n(0,0)\right\rangle=0$. Since $n^{\prime}(0,0) \neq(0,0)$ by Lemma $2.11,\left\{n, n^{\prime} /\left|n^{\prime}\right|\right\}$ forms an orthonormal basis for $\mathbb{R}^{2}$. Therefore,

$$
\sigma^{\prime \prime}(0,0)=\left\langle\sigma^{\prime \prime}(0,0), n^{\prime}(0,0)\right\rangle n^{\prime}(0,0) /\left|n^{\prime}(0,0)\right|^{2} .
$$

On the other hand, by differentiating (2-5) and by using Lemma 2.11, we have

$$
\varphi(0)=\varphi^{\prime}(0)=\varphi^{\prime \prime}(0)=0, \quad-\varphi^{\prime \prime \prime}(0)=\left\langle\sigma^{\prime \prime}(0,0), n^{\prime}(0,0)\right\rangle .
$$

Hence $\varphi$ has an $\mathscr{A}_{2}$-singularity at the origin if and only if $\left\langle\sigma^{\prime \prime}(0,0), n^{\prime}(0,0)\right\rangle \neq 0$. This and (2-6) prove the assertion.

Proposition 2.14. Suppose $(0,0)$ is a nondegenerate singular point. Then the germ of the image of the front is locally diffeomorphic to a cuspidal edge if and only if $(0,0)$ is of type $C$.

Proof. By Lemma 2.2, local diffeomorphic equivalence between singular points on fronts implies Legendrian equivalence. Since a cuspidal edge itself is of type C, any singular point locally diffeomorphic to a cuspidal edge is of type C. Conversely, we shall show that a singularity of type $\mathrm{C}$ is locally diffeomorphic to a cuspidal edge. To prove this, it is sufficient to show that $\varphi$ as above has an $\mathscr{A}_{2}$-singularity 
and $\Phi$ is versal. By Lemma 2.12 and (2-5), we have

$$
\left(\begin{array}{ll}
\Phi_{x}(0, \mathbf{0}) & \Phi_{x}^{\prime}(0, \mathbf{0}) \\
\Phi_{y}(0, \mathbf{0}) & \Phi_{y}^{\prime}(0, \mathbf{0}) \\
\Phi_{z}(0, \mathbf{0}) & \Phi_{z}^{\prime}(0, \mathbf{0})
\end{array}\right)=\left(\begin{array}{cc}
n_{1}(0,0) & n_{1}^{\prime}(0,0) \\
n_{2}(0,0) & n_{2}^{\prime}(0,0) \\
0 & 0
\end{array}\right) .
$$

This matrix is of rank 2 , since $n(0,0)$ and $n^{\prime}(0,0)$ are linearly independent.

Next we prove that $\varphi$ has an $\mathscr{A}_{2}$-singularity. We set

$$
\lambda=\operatorname{det}\left(\hat{f}_{w}, \hat{f}_{z}, \hat{v}\right),
$$

where $\hat{v}$ is the unit normal vector of the front $\hat{f}$. Here $\lambda=0$ on the singular curve. Since we have assumed that $(0,0)$ is of type C, Proposition 2.6(4) implies that $\partial / \partial z$ is the singular direction at the origin. So we have

$$
\lambda_{z}(0,0)=0 .
$$

On the other hand,

$$
0 \neq \lambda_{w}=\operatorname{det}\left(\hat{f}_{w w}, \hat{f}_{z}, \hat{v}\right)+\operatorname{det}\left(\hat{f}_{w}, \hat{f}_{z w}, \hat{v}\right)+\operatorname{det}\left(\hat{f}_{w}, \hat{f}_{z}, \hat{v}_{w}\right)=\operatorname{det}\left(\hat{f}_{w w}, \hat{f}_{z}, \hat{v}\right)
$$

holds at $(0,0)$, because $\hat{f}_{w}(0,0)=\mathbf{0}$. Since $\hat{f}_{z}(0,0) \times \hat{v}(0,0)$ is parallel to the $x y$-plane, we have

$$
\left\langle\sigma^{\prime \prime}(0,0), \hat{f}_{z}(0,0) \times \hat{v}(0,0)\right\rangle=\left\langle\hat{f}_{w w}(0,0), \hat{f}_{z}(0,0) \times \hat{v}(0,0)\right\rangle \neq 0 .
$$

In particular we have $\sigma^{\prime \prime}(0,0) \neq(0,0)$, and by Lemma $2.13, \varphi(w)$ has an $\mathscr{A}_{2^{-}}$ singularity at $w=0$.

Next, we prove the criterion for swallowtails:

Proposition 2.15. Suppose $(0,0)$ is a nondegenerate singular point. Then the germ of the image of the front is locally diffeomorphic to a swallowtail if and only if $(0,0)$ is of type $S$.

To prove this, we prepare a lemma:

Lemma 2.16. Suppose $(0,0)$ is a nondegenerate singular point of $\hat{f}(z, w)$, but not of type $C$. Then

(1) $\hat{f}_{w w}(0,0)=\mathbf{0}$, in particular $\sigma^{\prime \prime}(0,0)=(0,0)$, and

(2) $\Phi_{z}^{\prime \prime}(0,0,0,0) \neq 0$.

Proof. By (3) of Proposition 2.6, $\hat{f}_{w}$ vanishes identically on the singular curve. Since $(0,0)$ is not of type $\mathrm{C}$, the singular direction is equal to the null direction $\partial / \partial w$, thus $\hat{f}_{w w}(0,0)=\mathbf{0}$. In particular, we have $\sigma^{\prime \prime}(0,0)=(0,0)$. Differentiating (2-5) by $w$ and $z$ and substituting the relation

$$
\sigma(0,0)=\sigma^{\prime}(0,0)=\sigma_{z}(0,0)=\sigma^{\prime \prime}(0,0)=(0,0),
$$


we have

$$
-\Phi_{z}^{\prime \prime}(0,0,0,0)=\left\langle n^{\prime}(0,0), \sigma_{z}^{\prime}(0,0)\right\rangle .
$$

Since $\left\langle n, \sigma^{\prime}\right\rangle=0$, we have $\left\langle n, \sigma_{z}^{\prime}\right\rangle=0$. Since $n^{\prime}$ is orthogonal to $n, \sigma_{z}^{\prime}$ is proportional to $n^{\prime}$. To show $\Phi_{z}^{\prime \prime}(0,0,0,0) \neq 0$, it is sufficient to show $\sigma_{z}^{\prime}(0,0) \neq(0,0)$. Moreover, $\left(\hat{f}_{3}\right)_{w z}$ vanishes identically, so $\sigma_{z}^{\prime}(0,0) \neq(0,0)$ is equivalent to $\hat{f}_{z w}(0,0) \neq \mathbf{0}$.

Differentiating

$$
\lambda=\operatorname{det}\left(\hat{f}_{w}, \hat{f}_{z}, \hat{v}\right)
$$

with respect to $z$ and using the relation $\hat{f}_{w}(0,0)=\mathbf{0}$, we have

$$
\lambda_{z}(0,0)=\operatorname{det}\left(\hat{f}_{w z}(0,0), \hat{f}_{z}(0,0), \hat{v}(0,0)\right) .
$$

Since $(0,0)$ is not of type $\mathrm{C}, \partial / \partial w$ is the singular direction. In particular, $\lambda_{w}(0,0)=$ 0 . Since $d \lambda \neq 0$ at $(0,0)$, we have $\lambda_{z}(0,0) \neq 0$. Hence $\hat{f}_{z w}(0,0) \neq \mathbf{0}$ by $(2-7)$.

Proof of Proposition 2.15. For the same reason as in the proof of Proposition 2.14, any singular point locally diffeomorphic to a swallowtail is of type S. Conversely, we shall show that a singularity of type $S$ is locally diffeomorphic to a swallowtail. To prove this, it is sufficient to show that $\varphi$ as above has an $\mathscr{A}_{3}$-singularity and $\Phi$ is versal. By Lemma 2.12 and (2-5), we have

$$
\left(\begin{array}{lll}
\Phi_{x}(0, \mathbf{0}) & \Phi_{x}^{\prime}(0, \mathbf{0}) & \Phi_{x}^{\prime \prime}(0, \mathbf{0}) \\
\Phi_{y}(0, \mathbf{0}) & \Phi_{y}^{\prime}(0, \mathbf{0}) & \Phi_{y}^{\prime \prime}(0, \mathbf{0}) \\
\Phi_{z}(0, \mathbf{0}) & \Phi_{z}^{\prime}(0, \mathbf{0}) & \Phi_{z}^{\prime \prime}(0, \mathbf{0})
\end{array}\right)=\left(\begin{array}{ccc}
n_{1}(0,0) & n_{1}^{\prime}(0,0) & * \\
n_{2}(0,0) & n_{2}^{\prime}(0,0) & * \\
0 & 0 & \Phi_{z}^{\prime \prime}(0, \mathbf{0})
\end{array}\right) .
$$

By Lemma 2.16, $\Phi_{z}^{\prime \prime}(0, \mathbf{0}) \neq 0$, and then the rank of this matrix is 3. By Lemma 2.11 and Lemma 2.16, we have

$$
\varphi(0)=\varphi^{\prime}(0)=\varphi^{\prime \prime}(0)=\varphi^{\prime \prime \prime}(0)=0, \quad \varphi^{(4)}(0)=\left\langle\sigma^{\prime \prime \prime}(0), n^{\prime}(0)\right\rangle .
$$

By differentiating $\left\langle\sigma^{\prime}, n\right\rangle=0$ twice, we have

$$
\left\langle\sigma^{\prime \prime \prime}(0,0), n(0,0)\right\rangle=0 .
$$

Thus, $\varphi(w)$ has an $\mathscr{A}_{3}$-singularity if and only if $\sigma^{\prime \prime \prime}(0) \neq 0$, which is equivalent to $\hat{f}_{w w w}(0,0) \neq \mathbf{0}$, since $\hat{f}_{3}(z, w)=z$.

Since $\lambda(z, w)=\operatorname{det}\left(\hat{f}_{w}, \hat{f}_{z}, \hat{v}\right)$ and the singular curve $\gamma$ is given by $\lambda(z, w)=0$, the singular direction is given by

$$
\xi(z, w)=\left(\lambda_{w}(z, w),-\lambda_{z}(z, w)\right) \quad \text { on } \gamma .
$$

On the other hand, the null direction $\eta$ is given by

$$
\eta(z, w)=\frac{\partial}{\partial w} \quad \text { on } \gamma .
$$


Since $(0,0)$ is of type $S$, we have

$$
0 \neq\left.\frac{d}{d w}\right|_{w=0} \operatorname{det}(\xi(z, w), \eta(z, w))=\lambda_{w w}(0,0) .
$$

By the definition of $\lambda$, we have

$$
\lambda_{w}=\operatorname{det}\left(\hat{f}_{w w}, \hat{f}_{z}, \hat{v}\right)+\operatorname{det}\left(\hat{f}_{w}, \hat{f}_{z w}, \hat{v}\right)+\operatorname{det}\left(\hat{f}_{w}, \hat{f}_{z}, \hat{v}_{w}\right) .
$$

Here, since $\hat{f}_{w} \times \hat{f}_{z}$ is proportional to $\hat{v}$, we have

$$
\operatorname{det}\left(\hat{f}_{w}, \hat{f}_{z}, \hat{v}_{w}\right)=\left\langle\hat{f}_{w} \times \hat{f}_{z}, \hat{v}_{w}\right\rangle=0 .
$$

Thus, we have

$$
\lambda_{w}=\operatorname{det}\left(\hat{f}_{w w}, \hat{f}_{z}, \hat{v}\right)+\operatorname{det}\left(\hat{f}_{w}, \hat{f}_{z w}, \hat{v}\right)
$$

Then we get

$$
\begin{aligned}
\lambda_{w w}=\operatorname{det}\left(\hat{f}_{w w w}, \hat{f}_{z}, \hat{v}\right)+\operatorname{det}\left(\hat{f}_{w w}, \hat{f}_{z}, \hat{v}_{w}\right) \\
+2 \operatorname{det}\left(\hat{f}_{w w}, \hat{f}_{w z}, \hat{v}\right)+\operatorname{det}\left(\hat{f}_{w}, \hat{f}_{w w z}, \hat{v}\right)+\operatorname{det}\left(\hat{f}_{w}, \hat{f}_{w z}, \hat{v}_{w}\right) .
\end{aligned}
$$

Using $\hat{f}_{w}(0,0)=\hat{f}_{w w}(0,0)=\mathbf{0}$ and $(2-8)$, we have

$$
0 \neq \lambda_{w w}(0,0)=\operatorname{det}\left(\hat{f}_{w w w}(0,0), \hat{f}_{z}(0,0), \hat{v}(0,0)\right),
$$

which proves $\hat{f}_{w w w}(0,0) \neq \mathbf{0}$.

Izumiya and Takeuchi [2003] gave criteria for the singularities of a noncylindrical flat ruled front in the Euclidean 3 -space $\mathbb{R}^{3}$ to be cuspidal edges and swallowtails. One can prove those criteria by directly applying our criteria.

\section{Local properties of flat fronts in $H^{3}$}

In this section, we give a proof of Theorem 1.1 in the introduction, and show that, generically, singular points of flat fronts are cuspidal edges or swallowtails.

Preliminaries. We denote by $\boldsymbol{L}^{4}$ the Minkowski 4 -space with the inner product $\langle$,$\rangle of signature (-,+,+,+)$. The hyperbolic 3 -space $H^{3}$ is considered as the upper half component of the two sheet hyperboloid in $\boldsymbol{L}^{4}$ with the metric induced by $\langle$,$\rangle . Identifying \boldsymbol{L}^{4}$ with $\operatorname{Herm}(2)$, the set of $2 \times 2$-hermitian matrices, via

$$
\boldsymbol{L}^{4} \ni\left(x_{0}, x_{1}, x_{2}, x_{3}\right) \leftrightarrow\left(\begin{array}{cc}
x_{0}+x_{3} & x_{1}+\sqrt{-1} x_{2} \\
x_{1}-\sqrt{-1} x_{2} & x_{0}-x_{3}
\end{array}\right) \in \operatorname{Herm}(2),
$$


one has $\langle X, X\rangle=-\operatorname{det} X$ for $X \in \operatorname{Herm}(2)$, and $H^{3}$ is represented as

$$
\begin{aligned}
H^{3} & =\left\{x=\left(x_{0}, x_{1}, x_{2}, x_{3}\right) \in L^{4} ;\langle x, x\rangle=-1, x_{0}>0\right\} \\
& =\{X \in \operatorname{Herm}(2) ; \operatorname{det} X=1, \operatorname{trace} X>0\} \\
& =\left\{a a^{*} ; a \in \operatorname{SL}(2, \mathbb{C})\right\}=\operatorname{SL}(2, \mathbb{C}) / \operatorname{SU}(2) .
\end{aligned}
$$

The tangent space of $H^{3}$ at $p \in H^{3}$ is the set of vectors in $L^{4}$ perpendicular to $p$ :

$$
T_{p} H^{3}=\left\{Y \in L^{4} ;\langle p, Y\rangle=0\right\} .
$$

We define a bilinear, skew-symmetric product $\times$ as

$$
X \times Y:=\frac{\sqrt{-1}}{2}\left(X p^{-1} Y-Y p^{-1} X\right) \quad \text { for } \quad X, Y \in T_{p} H^{3},
$$

where $X, Y$ and $p$ are considered as matrices in $\operatorname{Herm}(2)$, and the products of the right-hand side are matrix multiplications. It is easy to show that $X \times Y$ is a vector in $T_{p} H^{3}$ and perpendicular to both $X$ and $Y$. We call " $\times$ " the cross product of $H^{3}$.

Let $M^{2}$ be an oriented simply-connected Riemannian 2-manifold, and let

$$
f: M^{2} \rightarrow H^{3}=\mathrm{SL}(2, \mathbb{C}) / \mathrm{SU}(2)
$$

be a front whose Legendrian lift is

$$
L_{f}: M^{2} \rightarrow T_{1}^{*} H^{3}=\mathrm{SL}(2, \mathbb{C}) / \mathrm{U}(1) .
$$

Identifying $T_{1}^{*} H^{3}$ with $T_{1} H^{3}$, we can write $L_{f}=(f, v)$, where $v(p)$ is a unit vector in $T_{p} H^{3}$ such that $\langle d f(p), v(p)\rangle=0$ for each $p \in M^{2}$. We call $v$ the unit vector field of the front $f$.

Suppose that $f$ is flat, then there is a (unique) complex structure on $M^{2}$ and a holomorphic Legendrian immersion

$$
E_{f}: M^{2} \rightarrow \operatorname{SL}(2, \mathbb{C})
$$

such that $f$ and $L_{f}$ are projections of $E_{f}$, where being a holomorphic Legendrian map means that $E_{f}^{-1} d E_{f}$ is off-diagonal. In particular, $f=E_{f} E_{f}^{*}$, with $H^{3}$ considered to be as in (1-2); see [Gálvez et al. 2000; Kokubu et al. 2003; 2004] for details. If we set

$$
E_{f}^{-1} d E_{f}=\left(\begin{array}{cc}
0 & \theta \\
\omega & 0
\end{array}\right)
$$

the first and the second fundamental forms $d s^{2}$ and $d h^{2}$ are given by

$$
\begin{aligned}
& d s^{2}=\omega \theta+\bar{\omega} \bar{\theta}+\left(|\omega|^{2}+|\theta|^{2}\right), \\
& d h^{2}=|\theta|^{2}-|\omega|^{2} .
\end{aligned}
$$


We call $\omega$ and $\theta$ the canonical forms of the front $f$. The holomorphic 2-differential

$$
Q:=\omega \theta,
$$

which appears in the $(2,0)$-part of $d s^{2}$, is called the Hopf differential of $f$. By definition, the umbilic points of the front $f$ coincide with the zeroes of $Q$. We remark that the $(1,1)$-part of the first fundamental form

$$
d s_{1,1}^{2}:=|\omega|^{2}+|\theta|^{2}
$$

is positive definite on $M^{2}$.

Conversely, the following assertion holds (see [Kokubu et al. 2004] for the front case and [Gálvez et al. 2000] for the regular case):

Fact 3.1. Let $\omega$ and $\theta$ be holomorphic 1-forms on a simply-connected Riemann surface $M^{2}$ such that $|\omega|^{2}+|\theta|^{2}$ is positive definite. Then the solution of the ordinary differential equation

$$
E^{-1} d E=\left(\begin{array}{cc}
0 & \theta \\
\omega & 0
\end{array}\right), \quad E\left(z_{0}\right)=\left(\begin{array}{ll}
1 & 0 \\
0 & 1
\end{array}\right)
$$

gives a holomorphic Legendrian immersion of $M^{2}$ into $\operatorname{SL}(2, \mathbb{C})$, where $z_{0} \in M^{2}$ is a base point, and its projection into $\mathrm{H}^{3}$ gives a flat front. Conversely, any flat front is locally expressed in this manner.

Remark 3.2. If we identify $H^{3}$ with the upper-half component of the hyperboloid in Minkowski 4-space $\boldsymbol{L}^{4}$, the parallel surface of $f$ is written as

$$
f_{t}=(\cosh t) f+(\sinh t) v: M^{2} \rightarrow H^{3} \subset \boldsymbol{L}^{4},
$$

where $t$ is the signed distance from $f$ and $v$ is the unit normal vector of $f$ in $H^{3}$. As pointed out in [Gálvez et al. 2000] and [Kokubu et al. 2004],

$$
E_{f_{t}}=E_{f}\left(\begin{array}{cc}
e^{t / 2} & 0 \\
0 & e^{-t / 2}
\end{array}\right) \text {. }
$$

Then the canonical forms $\omega_{t}$ and $\theta_{t}$ of $f_{t}$ are written as

$$
\omega_{t}=e^{t} \omega, \quad \theta_{t}=e^{-t} \theta .
$$

Proof of Theorem 1.1. Let $f: M^{2} \rightarrow H^{3}$ be a flat front. Then, on a neighborhood of $p$, we can take a holomorphic Legendrian immersion $E_{f}$ as in (3-2). Since $d s_{1,1}^{2}=|\omega|^{2}+|\theta|^{2}$ is positive definite, at least one of $\omega(p)$ or $\theta(p)$ is nonzero. So, by (3-2) and the fact that $f=E_{f} E_{f}^{*}$, we have

$$
f^{-1} d f=\left(E_{f}^{*}\right)^{-1}\left(\left(\begin{array}{cc}
0 & \theta \\
\omega & 0
\end{array}\right)+\left(\begin{array}{cc}
0 & \bar{\omega} \\
\bar{\theta} & 0
\end{array}\right)\right) E_{f}^{*} .
$$


Thus, if we write $\omega=\hat{\omega} d z$ and $\theta=\hat{\theta} d z$ in a complex coordinate $z$, we have

$$
f^{-1} f_{z}=\left(E_{f}^{*}\right)^{-1}\left(\begin{array}{cc}
0 & \hat{\theta} \\
\hat{\omega} & 0
\end{array}\right) E_{f}^{*} \quad \text { and } \quad f^{-1} f_{\bar{z}}=\left(E_{f}^{*}\right)^{-1}\left(\begin{array}{cc}
0 & \overline{\hat{\omega}} \\
\hat{\hat{\theta}} & 0
\end{array}\right) E_{f}^{*},
$$

and then

$$
\left(f^{-1} f_{z}\right) \times\left(f^{-1} f_{\bar{z}}\right)=\left(|\hat{\theta}|^{2}-|\hat{\omega}|^{2}\right)\left(\begin{array}{rr}
1 & 0 \\
0 & -1
\end{array}\right),
$$

where $\times$ is the cross product as in (3-1). Thus, the singular set is the set of zeroes of the function

$$
\lambda=|\hat{\theta}|^{2}-|\hat{\omega}|^{2} .
$$

Then $p$ is a singular point if and only if

$$
|\hat{\omega}(p)|=|\hat{\theta}(p)| .
$$

Hence (1) is proven. Since $f$ is a front, $d s_{1,1}^{2}$ as in (3-6) is positive definite. Hence $|\hat{\omega}(p)|=|\hat{\theta}(p)| \neq 0$ on a singular point $p$.

Moreover, at a singular point $p$, we have

$$
\begin{aligned}
d \lambda & =d(\hat{\theta} \overline{\hat{\theta}}-\hat{\omega} \overline{\hat{\omega}})=\left(\hat{\theta}^{\prime} \overline{\hat{\theta}}-\hat{\omega}^{\prime} \overline{\hat{\omega}}\right) d z+\left(\hat{\theta} \overline{\hat{\theta}}^{\prime}-\hat{\omega} \overline{\hat{\omega}}^{\prime}\right) d \bar{z} \\
& =\left(\hat{\theta}^{\prime} \frac{\hat{\theta} \overline{\hat{\theta}}}{\hat{\theta}}-\hat{\omega}^{\prime} \overline{\hat{\omega}}\right) d z+\overline{\left(\hat{\theta}^{\prime} \frac{\hat{\theta} \overline{\hat{\theta}}}{\hat{\theta}}-\hat{\omega}^{\prime} \overline{\hat{\omega}}\right)} d \bar{z} \\
& =\left(\hat{\theta}^{\prime} \frac{\hat{\omega} \overline{\hat{\omega}}}{\hat{\theta}}-\hat{\omega}^{\prime} \overline{\hat{\omega}}\right) d z+\overline{\left(\hat{\theta}^{\prime} \frac{\hat{\omega} \overline{\hat{\omega}}}{\hat{\theta}}-\hat{\omega}^{\prime} \overline{\hat{\omega}}\right)} d \bar{z} \\
& =\frac{\overline{\hat{\omega}}}{\hat{\hat{\theta}}}\left(\hat{\theta}^{\prime} \hat{\omega}-\hat{\omega}^{\prime} \hat{\theta}\right) d z+\frac{\hat{\omega}}{\overline{\hat{\theta}}} \overline{\left(\hat{\theta}^{\prime} \hat{\omega}-\hat{\omega}^{\prime} \hat{\theta}\right)} d \bar{z} .
\end{aligned}
$$

Hence a singular point $p$ is nondegenerate if and only if

$$
\hat{\theta}^{\prime} \hat{\omega}-\hat{\omega}^{\prime} \hat{\theta} \neq 0
$$

at $p$.

Let $p$ be a nondegenerate singular point, that is, (3-9) and (3-10) hold at $p$. Let $\gamma(t)$ be a singular curve such that $\gamma(0)=p$. Since $|\hat{\theta}|^{2}-|\hat{\omega}|^{2}=0$ on $\gamma(t)$,

$$
\begin{aligned}
0 & =\left(\hat{\theta}^{\prime} \overline{\hat{\theta}}-\hat{\omega}^{\prime} \overline{\hat{\omega}}\right) \dot{\gamma}+\overline{\left(\hat{\theta}^{\prime} \overline{\hat{\theta}}-\hat{\omega}^{\prime} \overline{\hat{\omega}}\right)} \dot{\bar{\gamma}} \\
& =|\hat{\theta}|^{2}\left(\frac{\hat{\theta}^{\prime}}{\hat{\theta}}-\frac{\hat{\omega}^{\prime}}{\hat{\omega}}\right) \dot{\gamma}+|\hat{\theta}|^{2} \overline{\left(\frac{\hat{\theta}^{\prime}}{\hat{\theta}}-\frac{\hat{\omega}^{\prime}}{\hat{\omega}}\right)} \dot{\bar{\gamma}}=2|\hat{\theta}|^{2}\left\langle\overline{\left(\frac{\hat{\theta}^{\prime}}{\hat{\theta}}-\frac{\hat{\omega}^{\prime}}{\hat{\omega}}\right)}, \dot{\gamma}\right\rangle
\end{aligned}
$$


holds on $\gamma(t)$, because of (3-9), where $\langle, \quad\rangle$ is the Hermitian inner product on $\mathbb{C}$. Here we identify $T_{p} M^{2}$ with $\mathbb{R}^{2}$ and $\mathbb{C}$ via the correspondence

$$
\zeta=a+\sqrt{-1} b \in \mathbb{C} \leftrightarrow(a, b) \in \mathbb{R}^{2} \leftrightarrow a \frac{\partial}{\partial u}+b \frac{\partial}{\partial v}=\zeta \frac{\partial}{\partial z}+\bar{\zeta} \frac{\partial}{\partial \bar{z}},
$$

where $z=u+\sqrt{-1} v$. Hence $\sqrt{-1} \overline{\left(\hat{\theta}^{\prime} / \hat{\theta}-\hat{\omega}^{\prime} / \hat{\omega}\right)}$ gives the singular direction. Thus, by a suitable choice of the parameter $t$, the singular curve $\gamma(t)$ can be parametrized as

$$
\dot{\gamma}(t)=\left.\sqrt{-1} \overline{\left(\frac{\hat{\theta}^{\prime}}{\hat{\theta}}-\frac{\hat{\omega}^{\prime}}{\hat{\omega}}\right)}\right|_{z=\gamma(t)} .
$$

The first fundamental form $d s^{2}$ is written as

$$
d s^{2}=(\hat{\omega} d z+\overline{\hat{\theta}} d \bar{z})(\hat{\theta} d z+\overline{\hat{\omega}} d \bar{z})
$$

on the curve $\gamma(t)$. Now we set $\rho=\hat{\theta} / \hat{\omega}$. Since $\rho(p) \neq 0$, there exists a holomorphic function $g$ defined on a neighborhood of $p$ such that $g^{2}=\rho$. Since $|g|=1$ on the singular curve $\gamma(t)$, we have

$$
\hat{\omega}\left(\frac{\sqrt{-1}}{g \hat{\omega}}\right)+\overline{\hat{\theta}} \overline{\left(\frac{\sqrt{-1}}{g \hat{\omega}}\right)}=\sqrt{-1}\left(\frac{1}{g}-\bar{g}\right)=0 .
$$

Thus the null direction is

$$
\eta(t)=\frac{\sqrt{-1}}{g \hat{\omega}}=\frac{\sqrt{-1}}{\sqrt{\hat{\omega} \hat{\theta}}} .
$$

So we have

$$
\operatorname{det}(\dot{\gamma}, \eta)=\operatorname{Im} \overline{\dot{\gamma}} \eta=\operatorname{Im}\left(\left(\frac{\hat{\theta}^{\prime}}{\hat{\theta}}-\frac{\hat{\omega}^{\prime}}{\hat{\omega}}\right) \frac{1}{\sqrt{\hat{\omega} \hat{\theta}}}\right) .
$$

Here, by Proposition 1.3, $p$ is diffeomorphic to a cuspidal edge if $\operatorname{det}(\dot{\gamma}, \eta) \neq 0$ at $t=0$. Hence, we have (2).

Next we prove (3). Using (3-11) and (3-12), we can compute that

$$
\frac{d}{d t} \operatorname{det}(\dot{\gamma}, \eta)=\operatorname{Re}\left(\frac{s(\hat{\theta})-s(\hat{\omega})}{\sqrt{\hat{\omega} \hat{\theta}}}|\hat{\omega} \hat{\theta}| \overline{\left(\frac{\hat{\theta}^{\prime} / \hat{\theta}-\hat{\omega}^{\prime} / \hat{\omega}}{\sqrt{\hat{\omega} \hat{\theta}}}\right)}\right) .
$$

Hence, if $\left(\hat{\theta}^{\prime} / \hat{\theta}-\hat{\omega}^{\prime} / \hat{\omega}\right) / \sqrt{\hat{\omega} \hat{\theta}} \in \mathbb{R}$ at $p$, then

$$
\left.\frac{d}{d t} \operatorname{det}(\dot{\gamma}, \eta)\right|_{t=0}=\left.\left(|\hat{\omega} \hat{\theta}| \frac{\hat{\theta}^{\prime} / \hat{\theta}-\hat{\omega}^{\prime} / \hat{\omega}}{\sqrt{\hat{\omega} \hat{\theta}}} \operatorname{Re} \frac{s(\hat{\theta})-s(\hat{\omega})}{\sqrt{\hat{\omega} \hat{\theta}}}\right)\right|_{p} .
$$


This proves (3), because of Proposition 1.3.

Remark 3.3. We set the two hyperbolic Gauss maps to be

$$
G=\frac{A}{C}, \quad G_{*}=\frac{B}{D}, \quad \text { where } E_{f}=\left(\begin{array}{cc}
A & B \\
C & D
\end{array}\right) .
$$

As shown in [Kokubu et al. 2003], we have the following expression

$$
E_{f}=\left(\begin{array}{cc}
G / \Delta & \Delta G_{*} /\left(G-G_{*}\right) \\
1 / \Delta & \Delta /\left(G-G_{*}\right)
\end{array}\right), \quad \Delta:=c \exp \left(\int_{z_{0}}^{z} \frac{d G}{G-G_{*}}\right),
$$

where $c=e^{-t / 2} \in \mathbb{R}$ determines which member of the parallel family $f_{t}$ of $f$ we have. In this $\left(G, G_{*}\right)$-construction of flat fronts, it is convenient to rewrite the conditions in Theorem 1.1 in terms of $\left(G, G_{*}\right)$. We have the following identities, which will be useful for an application of Theorem 1.1 (see [Kokubu et al. 2004]):

$$
\begin{aligned}
& Q:=\omega \theta=-\frac{d G d G_{*}}{\left(G-G_{*}\right)^{2}}, \quad \omega=-\Delta^{-2} d G, \quad \theta=\frac{\Delta^{2} d G_{*}}{\left(G-G_{*}\right)^{2}}, \\
& \frac{\hat{\omega}^{\prime}}{\hat{\omega}}=\frac{G^{\prime \prime}}{G^{\prime}}-2 \frac{G^{\prime}}{G-G_{*}}, \quad \frac{\hat{\theta}^{\prime}}{\hat{\theta}}=\frac{G_{*}^{\prime \prime}}{G_{*}^{\prime}}-2 \frac{G_{*}^{\prime}}{G_{*}-G}, \\
& s(\hat{\omega})=2 \hat{Q}+\{G, z\}, \quad s(\hat{\theta})=2 \hat{Q}+\left\{G_{*}, z\right\},
\end{aligned}
$$

where ' $=d / d z, Q=\hat{Q} d z^{2}, s(\cdot)$ is as in (1-3) and $\{G, z\}$ denotes the Schwarzian derivative of $G$ with respect to $z$ :

$$
\{G, z\}=\left(\frac{G^{\prime \prime}}{G^{\prime}}\right)^{\prime}-\frac{1}{2}\left(\frac{G^{\prime \prime}}{G^{\prime}}\right)^{2} .
$$

Generic singularities of flat fronts. As an application of Theorem 1.1, we shall now show that generic singularities of flat fronts are cuspidal edges or swallowtails. Let $U$ be a simply-connected domain in $\mathbb{C}$, and $\mathbb{O}(U)$ the set of holomorphic functions on $U$. Then, for each $h \in \mathcal{O}(U)$, we can construct a flat front

$$
f_{h}: U \rightarrow H^{3}
$$

which is represented by a pair of holomorphic 1-forms $(\omega, \theta)=\left(d z, e^{h} d z\right)$.

Conversely, if $p$ is not an umbilic point (i.e. $Q(p)=\omega(p) \theta(p) \neq 0)$, both $\omega(p)$ and $\theta(p)$ are not equal to zero, and we can choose a complex coordinate such that $\omega=d z$ and $\theta=e^{h} d z$. Thus, any flat front is locally congruent to some $f_{h}$, except in neighborhoods of umbilic points. We remark that an umbilic point cannot be a singular point, since $\theta / \omega$ takes the value 0 or $\infty$ at umbilic points. By Theorem 1.1, we have: 
(1) The zeroes of $\operatorname{Re} h$ correspond to singular sets. Moreover, a singular point $p \in U$ is nondegenerate if and only if $h^{\prime}(p) \neq 0$.

(2) A singular point $p$ is a cuspidal edge if and only if it is nondegenerate and $e^{-h(p) / 2} h^{\prime}(p) \notin \mathbb{R}$.

(3) A singular point $p$ is a swallowtail if and only if it is nondegenerate and we have

$$
e^{-h(p) / 2} h^{\prime}(p) \in \mathbb{R} \quad \text { and } \quad \operatorname{Re}\left(e^{-h(p)}\left(h^{\prime \prime}(p)-\frac{1}{2} h^{\prime}(p)^{2}\right)\right) \neq 0 .
$$

We let $J_{H}^{k}(U)$ be the space of $k$-jets of holomorphic functions on $U$. Then $J_{H}^{k}(U)$ is canonically identified with the product space $U \times \mathbb{C}^{k+1}$ :

$$
J_{H}^{k}(U) \ni j^{k} h \longleftrightarrow\left(p, h(p), h^{\prime}(p), h^{\prime \prime}(p), \ldots, h^{(k)}(p)\right) \in U \times \mathbb{C}^{k+1} .
$$

In particular, $J_{H}^{k}(U)$ can be considered as a $C^{\infty}$-manifold of dimension $2(k+2)$ as well as a complex manifold of dimension $k+2$. For a compact set $K$ of $U$ and an open subset $O$ in $J_{H}^{k}(U)$, we set

$$
[K, O]_{k}:=\left\{h \in \mathbb{O}(U) ; j^{k} h(K) \subset O\right\} .
$$

Let $\mathfrak{O}_{k}$ be the topology of $\mathcal{O}(U)$ generated by such $[K, O]_{k}$, which is called the compact open $C^{k}$-topology. If $\pi: J_{H}^{k+1}(U) \rightarrow J_{H}^{k}(U)$ is the canonical projection, it can be easily seen that $\pi$ is a continuous map and $\left[K, \pi^{-1}(O)\right]_{k+1}=[K, O]_{k}$. In particular, $\mathfrak{O}_{k} \subset \mathfrak{O}_{k+1}$, and

$$
\mathfrak{O}:=\bigcup_{k=0}^{\infty} \mathfrak{O}_{k}
$$

gives a topology on $\mathcal{O}(U)$, called the compact open $C^{\infty}$-topology. A holomorphic function $h \in \mathbb{O}(U)$ is an interior point of a given subset $\mathscr{V}(\subset \mathbb{O}(U)$ ) (with respect to the compact open $C^{\infty}$-topology) if and only if there exist a nonnegative integer $l$ and a finite number of compact sets $C_{1}, \ldots, C_{s}$ in $U$ and open subsets $O_{1}, \ldots, O_{s} \subset J_{H}^{l}(U)$ such that

$$
h \in \bigcap_{r=1}^{s}\left[C_{r}, O_{r}\right]_{l} \subset \mathcal{V} .
$$

Now we give a topology on the family of flat fronts $\left\{f_{h}\right\}_{h \in \mathscr{O}(U)}$ induced from the compact open $C^{\infty}$-topology on $\mathbb{O}(U)$. We shall prove:

Theorem 3.4. Let $K$ be an arbitrary compact set of $U$ and $S(K)$ the subset of $\left\{f_{h}\right\}_{h \in \mathbb{O}(U)}$ which consists of $f_{h}$ whose singular points on $K$ are locally diffeomorphic to cuspidal edges or swallowtails. Then $S(K)$ is an open and dense subset of $\left\{f_{h}\right\}_{h \in \mathcal{O}(U)}$. 
Remark 3.5. Generic properties of $C^{\infty}$-maps are usually described in terms of the Whitney $C^{\infty}$-topology (see [Golubitsky and Guillemin 1973] for definition), because it is suitable for the technique of multiplying by a cut-off function. However, generic properties of analytic functions are different in the Whitney $C^{\infty}$ topology. In the theorem above, we use the compact open $C^{\infty}$-topology. These two topologies are the same when the source space is compact, but they differ on $\mathcal{O}(U)$. In fact, when the source space is noncompact, the compact open $C^{\infty}$ topology satisfies the second countability axiom, but the Whitney $C^{\infty}$-topology on $\mathcal{O}(U)$ does not satisfy even the first countability axiom and cannot be treated by sequence convergence. We do not know if the set $S(U)$ (which consists of $f_{h}$ whose singular points on $U$ are locally diffeomorphic to cuspidal edges or swallowtails) is an open dense subset with respect to the Whitney $C^{\infty}$-topology.

Proof of Theorem 3.4. We set

$$
\begin{aligned}
& A_{1}:=\left\{j^{2} h(p) \in J_{H}^{2}(U) \mid \operatorname{Re} h(p)=0 \text { and } h^{\prime}(p)=0\right\}, \\
& A_{2}:=\left\{\begin{array}{l|l}
j^{2} h(p) \in J_{H}^{2}(U) & \begin{array}{l}
\operatorname{Re} h(p)=0, \quad \operatorname{Im}\left(e^{-h(p) / 2} h^{\prime}(p)\right)=0, \\
\operatorname{Re}\left(e^{-h(p)}\left(h^{\prime \prime}(p)-\frac{1}{2} h^{\prime}(p)^{2}\right)\right)=0
\end{array}
\end{array}\right\} .
\end{aligned}
$$

Then $A_{1}$ and $A_{2}$ are both closed subsets of $J_{H}^{2}(U)$. The set

$$
\hat{S}(K)=\left\{h \in \mathcal{O}(U) ; j^{2} h(K) \subset J_{H}^{2}(U) \backslash\left(A_{1} \cup A_{2}\right)\right\}=\left[K, J_{H}^{2}(U) \backslash\left(A_{1} \cup A_{2}\right)\right]_{2}
$$

corresponds to $S(K)$ under the identification $h \leftrightarrow f_{h}$, which is by definition an open subset in $O(U)$.

So it is sufficient to show that $\hat{S}(K)$ is a dense subset. Obviously, $A_{1}$ is a real closed submanifold of $J_{H}^{2}(U)$ with codimension three. We remark that $J_{H}^{2}(U) \backslash A_{1}$ is an open submanifold of $J_{H}^{2}(U)$.

Lemma 3.6. $A_{2} \backslash A_{1}$ is a submanifold of $J_{H}^{2}(U)$ with codimension three.

Proof. We define a $C^{\infty}$-map $\zeta=\left(\zeta^{1}, \zeta^{2}, \zeta^{3}\right): J_{H}^{2}(U) \backslash A_{1} \rightarrow \mathbb{R}^{3}$ by

$$
\begin{aligned}
& \zeta\left(j^{2} h(p)\right)=\left(\operatorname{Re} h(p), \operatorname{Im}\left(e^{-h(p) / 2} h^{\prime}(p)\right), \operatorname{Re}\left(e^{-h(p)}\left(h^{\prime \prime}(p)-\frac{1}{2} h^{\prime}(p)^{2}\right)\right)\right) \\
&=\left(u, e^{-u / 2}\left(v_{1} \cos (v / 2)-u_{1} \sin (v / 2)\right),\right. \\
&\left.\frac{1}{2} e^{-u}\left(\left(-u_{1}^{2}+v_{1}^{2}+2 u_{2}\right) \cos v+2\left(-u_{1} v_{1}+v_{2}\right) \sin v\right)\right),
\end{aligned}
$$

where we set

$h(z)=u(z)+\sqrt{-1} v(z), h^{\prime}(z)=u_{1}(z)+\sqrt{-1} v_{1}(z), h^{\prime \prime}(z)=u_{2}(z)+\sqrt{-1} v_{2}(z)$.

Then $\left(z, u, v, u_{1}, v_{1}, u_{2}, v_{2}\right)$ gives the canonical coordinate system on $J_{H}^{2}(U)$. By a direct calculation, we have $\zeta^{-1}(0,0,0)=A_{2} \backslash A_{1}$. We show that $(0,0,0)$ is a 
regular value of $\zeta$. To determine the rank of the Jacobian matrix of $\zeta$ at any point in $\zeta^{-1}(0,0,0)$, we calculate the derivative of $\zeta$ with respect to $u, u_{1}$ and $v_{1}$ :

$$
\begin{aligned}
\left(\zeta_{u}^{1}, \zeta_{u}^{2}, \zeta_{u}^{3}\right) & =\left(1, \frac{1}{2} e^{-u / 2}\left(-v_{1} \cos (v / 2)+u_{1} \sin (v / 2)\right),\right. \\
& \left.-\frac{1}{2} e^{-u}\left(\left(-u_{1}^{2}+v_{1}^{2}+2 u_{2}\right) \cos v+2\left(-u_{1} v_{1}+v_{2}\right) \sin v\right)\right), \\
\left(\zeta_{u_{1}}^{1}, \zeta_{u_{1}}^{2}, \zeta_{u_{1}}^{3}\right) & =\left(0,-e^{-u / 2} \sin (v / 2),-e^{-u}\left(u_{1} \cos v+v_{1} \sin v\right)\right), \\
\left(\zeta_{v_{1}}^{1}, \zeta_{v_{1}}^{2}, \zeta_{v_{1}}^{3}\right) & =\left(0, e^{-u / 2} \cos (v / 2), e^{-u}\left(v_{1} \cos v-u_{1} \sin v\right)\right) .
\end{aligned}
$$

Then we have

$$
\frac{\partial\left(\zeta^{1}, \zeta^{2}, \zeta^{3}\right)}{\partial\left(u, u_{1}, v_{1}\right)}=e^{-3 u / 2}\left(u_{1} \cos (v / 2)+v_{1} \sin (v / 2)\right) .
$$

We now suppose $\zeta\left(z, u, v, u_{1}, v_{1}, u_{2}, v_{2}\right)=0$. Then

$$
u=0, \quad e^{-u / 2}\left(v_{1} \cos (v / 2)-u_{1} \sin (v / 2)\right)=0
$$

hold and thus

$$
v_{1} \cos \frac{v}{2}-u_{1} \sin \frac{v}{2}=0 .
$$

Then (3-18) and $\partial\left(\zeta^{1}, \zeta^{2}, \zeta^{3}\right) / \partial\left(u, u_{1}, v_{1}\right)=0$ imply that $u=u_{1}=v_{1}=0$, namely, that $\left(z, u, v, u_{1}, v_{1}, u_{2}, v_{2}\right)$ belongs to $A_{1}$. Hence $d \zeta$ is of rank 3 at $\zeta^{-1}(0,0,0)$ in $J_{H}^{2}(U) \backslash A_{1}$. By the implicit function theorem, $A_{2} \backslash A_{1}$ is a submanifold of codimension 3 .

We continue with the proof of Theorem 3.4. We next show that $\hat{S}(K)$ is a dense subset. Fix a function $h \in \mathbb{O}(U)$. Let $B$ be the set of polynomials of degree at most 2 in $z$ and define a map

$$
G: U \times B \ni(z, \varphi) \mapsto j^{2}(h+\varphi)(z) \in J_{H}^{2}(U) .
$$

Obviously the map $G$ is a diffeomorphism. Therefore $G^{-1}\left(A_{1}\right)$ and $G^{-1}\left(A_{2} \backslash A_{1}\right)$ are submanifolds of dimension 5 diffeomorphic to $A_{1}$ and $A_{2} \backslash A_{1}$ respectively. Let

$$
\pi: U \times B \rightarrow B
$$

be the canonical projection. Since $B$ is a $C^{\infty}$-manifold of dimension 6 , Sard's theorem yields that $\pi\left(G^{-1}\left(A_{1}\right)\right)$ and $\pi\left(G^{-1}\left(A_{2} \backslash A_{1}\right)\right)$ are measure zero sets in $B$. Thus

$\pi\left(G^{-1}\left(A_{1} \cup A_{2}\right)\right)=\pi\left(G^{-1}\left(A_{1} \cup\left(A_{2} \backslash A_{1}\right)\right)\right)=\pi\left(G^{-1}\left(A_{1}\right)\right) \cup \pi\left(G^{-1}\left(A_{2} \backslash A_{1}\right)\right)$

is also a measure zero set. Thus there is a sequence $\left\{\varphi_{n}\right\}$ in $B$ such that $\varphi_{n}$ converges to the zero polynomial and $\varphi_{n} \notin \pi\left(G^{-1}\left(A_{1} \cup A_{2}\right)\right)$. We set $h_{n}:=h+\varphi_{n}$. Then 
$\left(j^{2} h_{n}\right)(U) \notin A_{1} \cup A_{2}$, that is,

$$
h_{n} \in\left[U, J_{H}^{2}(U) \backslash\left(A_{1} \cup A_{2}\right)\right]_{2} \subset \hat{S}(K) .
$$

Let $d$ be a distance function on $J_{H}^{l}(U)$ which is compatible with respect to its topology. Then a sequence $\left\{g_{n}\right\}$ in $C^{0}\left(U, J_{H}^{l}(U)\right)$ converges to $g$ uniformly on a given compact subset $K$ of $U$ if for any $\varepsilon>0$, there exists a positive integer $n_{0}$ such that

$$
\sup _{z \in K} d\left(g_{n}(z), g(z)\right)<\varepsilon \quad\left(n \geq n_{0}\right) .
$$

We remark that

$$
j^{k}(\mathcal{O}(U)) \subset C^{0}\left(U, J_{H}^{l}(U)\right) .
$$

Since the difference $h_{n}-h$ is only a polynomial $\varphi_{n}$ of degree at most 2 converging to the zero polynomial, one can easily check that for each nonnegative integer $l$, $j^{l} h_{n}$ converges to $j^{l} h$ uniformly on any compact subset of $U$.

Let $\mathscr{V}$ be an open neighborhood of $h$ in $\mathcal{O}(U)$. Then by the definition of the compact open $C^{\infty}$-topology, there exist a nonnegative integer $l$, a finite number of compact sets $C_{1}, \ldots, C_{s}$ of $U$, and open subsets $O_{1}, \ldots, O_{s}$ of $J_{H}^{l}(U)$ such that

$$
h \in \bigcap_{r=1}^{s}\left[C_{r}, O_{r}\right]_{l} \subset \mathcal{V} .
$$

We set

$$
\Delta_{r}=d\left(j^{l} h\left(C_{r}\right), J_{H}^{l}(U) \backslash O_{r}\right)>0 \quad(r=1,2,3, \ldots, s) .
$$

Note that

$$
C:=C_{1} \cup C_{2} \cup \cdots \cup C_{s}
$$

is a compact set. Since $j^{l} h_{n}$ converges to $j^{l} h$ uniformly on any compact subset of $U$, there exists an integer $n_{0}>0$ such that

$$
\sup _{z \in C} d\left(\left(j^{l} h\right)(z),\left(j^{l} h_{n}\right)(z)\right)<\frac{1}{2} \min \left(\Delta_{1}, \ldots, \Delta_{s}\right) \quad\left(n \geq n_{0}\right) .
$$

On the other hand,

$d\left(\left(j^{l} h\right)(z), J_{H}^{l}(U) \backslash O_{r}\right) \leq d\left(\left(j^{l} h\right)(z),\left(j^{l} h_{n}\right)(z)\right)+d\left(j^{l}\left(h_{n}\right)(z), J_{H}^{l}(U) \backslash O_{r}\right)$, then

$$
\begin{aligned}
d\left(j^{l}\left(h_{n}\right)(z), J_{H}^{l}(U) \backslash O_{r}\right) & \geq d\left(j^{l} h(z), J_{H}^{l}(U) \backslash O_{r}\right)-d\left(\left(j^{l} h\right)(z),\left(j^{l} h_{n}\right)(z)\right) \\
& \geq \Delta_{r}-\frac{1}{2} \min \left(\Delta_{1}, \ldots, \Delta_{s}\right)>0 \quad\left(z \in C_{r}\right) .
\end{aligned}
$$


This implies that $j^{l} h_{n}(z) \in O_{r}$ if $z \in C_{r}$. Thus $h_{n} \in\left[C_{r}, O_{r}\right]_{l}$ for all $r=1,2, \ldots, s$, and

$$
h_{n} \in \bigcap_{r=1}^{s}\left[C_{r}, O_{r}\right]_{l} \subset \mathcal{V} \quad\left(n \geq n_{0}\right) .
$$

Since $h_{n} \in \hat{S}(K)$, this implies that $\hat{S}(K)$ is a dense subset.

\section{Global properties of singular points}

In this section, we shall give a proof of Theorem 1.2.

Preliminaries. Let $f: M^{2} \rightarrow H^{3}$ be a flat front defined on a Riemann surface $M^{2}$. In this section, we do not necessarily assume that $M^{2}$ is simply-connected. Thus the holomorphic lift $E_{f}$ of $f$ is defined only on the universal cover $\tilde{M}^{2}$ of $M^{2}$ :

$$
E_{f}: \tilde{M}^{2} \rightarrow \operatorname{SL}(2, \mathbb{C}),
$$

and then the canonical forms $\omega$ and $\theta$ as in (3-3) are holomorphic 1-forms defined on $\widetilde{M}^{2}$. Note that the first and second fundamental forms as in (3-4), the Hopf differential as in (3-5), and the $(1,1)$-part $d s_{1,1}^{2}$ of the first fundamental form are all well-defined on $M^{2}$. Moreover $d s_{1,1}^{2}$ is positive definite on $M^{2}$. We have that

$\omega$ and $\theta$ have no common zeroes on $\tilde{M}^{2}$, and

$$
|\omega|^{2} \text { and }|\theta|^{2} \text { are well-defined pseudometrics on } M^{2} \text {. }
$$

From now on, we assume $f$ is complete, that is, there exist a compact set $C \subset M^{2}$ and a symmetric 2-tensor $T$ such that $T$ is identically zero outside $C$ and $d s^{2}+T$ is a complete Riemannian metric; see [Kokubu et al. 2004]. We remark that $f$ is complete if and only if (see [Kokubu et al. 2005])

(1) the (1,1)-part $d s_{1,1}^{2}$ of the first fundamental form is complete (in this case, we say that $f$ is weakly complete),

(2) $d s_{1,1}^{2}$ has finite total absolute curvature, and

(3) the singular set is a compact set of $M^{2}$.

In the proof of Theorem 1.2, we use only properties (1) and (2); that is, the conclusion of Theorem 1.2 holds for weakly complete flat fronts such that $d s_{1,1}^{2}$ has finite absolute total curvature.

By completeness, we know that there exist a compact Riemann surface $\bar{M}^{2}$ and a finite number of points $\left\{p_{1}, \ldots, p_{N}\right\}$ in $\bar{M}^{2}$ such that

$$
M^{2} \cong \bar{M}^{2} \backslash\left\{p_{1}, \ldots, p_{N}\right\} \quad \text { (biholomorphism); }
$$

see [Kokubu et al. 2004, Lemma 3.3], and also [Gálvez et al. 2000]. We call the points $\left\{p_{j}\right\}$ the ends of $f$. Moreover, as shown in [Gálvez et al. 2000, Lemma 2], 
the Hopf differential $Q$ can be extended meromorphically on $\bar{M}^{2}$, and at each end $p_{j}$ there exists a complex coordinate $z$ around $p_{j}$ such that $z\left(p_{j}\right)=0$ and the canonical forms are written as

$$
\text { (4-3) } \quad \omega=\hat{\omega}(z) d z=z^{\mu} \hat{\omega}_{0}(z) d z, \quad \theta=\hat{\theta}(z) d z=z^{\mu_{*}} \hat{\theta}_{0}(z) d z \quad\left(\mu, \mu_{*} \in \mathbb{R}\right),
$$

where $\hat{\omega}_{0}$ and $\hat{\theta}_{0}$ are holomorphic functions in $z$ which do not vanish at the origin. Since $\mu$ and $\mu_{*}$ do not depend on the choice of complex coordinates, we denote

$$
\operatorname{ord}_{p_{j}} \omega:=\mu, \quad \operatorname{ord}_{p_{j}} \theta:=\mu_{*} .
$$

These are the orders of the pseudometrics $|\omega|^{2}$ and $|\theta|^{2}$, respectively. By (3-5), we have

$$
\mu+\mu_{*}=\operatorname{ord}_{p_{j}} \omega+\operatorname{ord}_{p_{j}} \theta=\operatorname{ord}_{p_{j}} Q \in \mathbb{Z},
$$

where, by convention, $\operatorname{ord}_{0} Q=k$ if $Q=z^{k} d z^{2}$. Since $f$ is complete, $d s_{1,1}^{2}$ is a complete Riemannian metric on $M^{2}$ [Kokubu et al. 2004, Corollary 3.4]. Thus,

$$
\min \left\{\operatorname{ord}_{p_{j}} \omega, \operatorname{ord}_{p_{j}} \theta\right\} \leq-1 .
$$

Definition 4.1. An end $p_{j}$ is called cylindrical if

$$
\operatorname{ord}_{p_{j}} \omega=\operatorname{ord}_{p_{j}} \theta .
$$

Let $G$ and $G_{*}$ be the hyperbolic Gauss maps of $f$. Then $G$ and $G_{*}$ are both meromorphic functions on $M^{2}$, and $G(p) \neq G_{*}(p)$ for all $p \in M^{2}$.

Fact 4.2 [Gálvez et al. 2000, Theorem 4]. At an end $p_{j}$, the following properties are equivalent:

(1) $G$ is meromorphic at $p_{j}$.

(2) $G_{*}$ is meromorphic at $p_{j}$.

(3) $\operatorname{ord}_{p_{j}} Q \geq-2$, that is, $Q$ has at most a pole of order 2 at $p_{j}$.

Definition 4.3. An end $p_{j}$ is called regular if the three properties in Fact 4.2 hold. Otherwise, $p_{j}$ is called irregular.

Remark 4.4. The ends of hyperbolic cylinders are regular and cylindrical. As a special case of [Gálvez et al. 2000, Theorem 6], a regular cylindrical end is asymptotic to a finite cover of a hyperbolic cylinder.

An umbilic point $q \in M^{2}$ is a zero of the Hopf differential $Q$. When $Q$ is identically zero, that is, $f$ is totally umbilic, $f$ represents the horosphere. In this section, we assume that $f$ is not totally umbilic. Since $Q$ is meromorphic on the 
compact Riemann surface $\bar{M}^{2}$, the number of umbilic points is finite. As $d s_{1,1}^{2}$ is positive definite at $q,(3-5)$ implies that

$$
\begin{aligned}
& \text { either } \operatorname{ord}_{q} \omega=\operatorname{ord}_{q} Q \in \mathbb{Z}_{+} \text {and } \operatorname{ord}_{q} \theta=0 \\
& \text { or } \quad \operatorname{ord}_{q} \theta=\operatorname{ord}_{q} Q \in \mathbb{Z}_{+} \text {and } \operatorname{ord}_{q} \omega=0
\end{aligned}
$$

at each umbilic point $q$.

Using a local complex coordinate $z$, we write

$$
\omega=\hat{\omega} d z, \quad \theta=\hat{\theta} d z, \quad Q=\hat{Q} d z^{2} .
$$

Global descriptions of the criteria for singular points. Let $\bar{M}^{2}$ be a compact Riemann surface and

$$
f: M^{2}=\bar{M}^{2} \backslash\left\{p_{1}, \ldots, p_{N}\right\} \rightarrow H^{3}
$$

a complete flat front which is not totally umbilic. Using the canonical forms $\omega$ and $\theta$ in (3-3), we define

$$
\rho:=\frac{\theta}{\omega} .
$$

Though $\rho$ might be defined only on the universal cover of $M^{2}$, (4-2) implies that $|\rho|$ is well-defined on $M^{2}$. Moreover, by (4-3), $|\rho|$ can be extended on $\bar{M}^{2}$ as a continuous map

$$
|\rho|: \bar{M}^{2} \rightarrow[0,+\infty] .
$$

As seen in Section 3, the set of singular points of the flat front $f$ is given by

$$
\Sigma(f):=\left\{p \in M^{2} ;|\rho(p)|=1\right\} .
$$

Using a local expression as in (4-6), we define

(4-7) $\xi:=\left(\frac{\hat{\theta}^{\prime}}{\hat{\theta}}-\frac{\hat{\omega}^{\prime}}{\hat{\omega}}\right) \hat{Q} d z^{3}, \quad \zeta_{c}:=\left(\frac{\hat{\theta}^{\prime}}{\hat{\theta}}-\frac{\hat{\omega}^{\prime}}{\hat{\omega}}\right)^{2} \frac{1}{\hat{Q}}, \quad$ and $\quad \zeta_{s}:=\frac{s(\hat{\theta})-s(\hat{\omega})}{\hat{Q}}$, where $Q=\hat{Q} d z^{2},{ }^{\prime}=d / d z$ and $s(\cdot)$ is as in (1-3).

Lemma 4.5. The quantities in (4-7) are independent of the choice of complex coordinates. In particular, $\xi$ is a meromorphic 3-differential on $\bar{M}^{2}$, and both $\zeta_{c}$ and $\zeta_{s}$ are meromorphic functions on $\bar{M}^{2}$.

Proof. Since $|\rho|$ is well-defined on $M^{2}$,

$$
d(\rho \bar{\rho})=|\rho|^{2}\left(\frac{d \rho}{\rho}+\frac{d \bar{\rho}}{\bar{\rho}}\right)
$$

is well-defined on $M^{2}$, and then so is its $(1,0)$-part. Hence

$$
\frac{d \rho}{\rho}=\left(\frac{\hat{\theta}^{\prime}}{\hat{\theta}}-\frac{\hat{\omega}^{\prime}}{\hat{\omega}}\right) d z
$$


is a meromorphic 1-form on $M^{2}$. Also, by (4-3) and (4-5), $d \rho / \rho$ is a meromorphic 1-form on $\bar{M}^{2}$. Since $Q$ is a meromorphic 2-differential on $\bar{M}^{2}, \xi=(d \rho / \rho) Q$ is a meromorphic 3-differential. As the symmetric product $(d \rho / \rho)(d \rho / \rho)$ is a meromorphic 2-differential,

$$
\zeta_{c}=\frac{(d \rho / \rho)(d \rho / \rho)}{Q}
$$

is a meromorphic function on $\bar{M}^{2}$.

Though the Schwarzian derivative as in (3-17) depends on the choice of complex coordinates, the difference of two Schwarzian derivatives is considered as a meromorphic 2-differential; that is, if we write $S(G):=\{G, z\} d z^{2}$ in the complex coordinate $z$

$$
S\left(G_{*}\right)-S(G)=\left(\left\{G_{*}, z\right\}-\{G, z\}\right) d z^{2}
$$

is independent of the choice of a coordinate $z$, as a meromorphic 2-differential. Here, by (3-16), we have

$$
\zeta_{s}=\frac{\left\{G_{*}, z\right\}-\{G, z\}}{\hat{Q}}=\frac{S\left(G_{*}\right)-S(G)}{Q} .
$$

This shows that $\zeta_{s}$ is a well-defined meromorphic function on $M^{2}$. Moreover, by (4-3) and the definition (4-7), $\zeta_{s}$ is meromorphic at each end.

Using the invariants of (4-7), we define

$$
\begin{aligned}
\Sigma(f) & :=\left\{p \in M^{2} ;|\rho(p)|=1\right\}, \\
Z_{0}(f) & :=\left\{p \in M^{2} ; \xi(p)=0\right\}, \\
Z_{c}(f) & :=\left\{p \in M^{2} ; \operatorname{Im} \sqrt{\zeta_{c}(p)}=0\right\}, \\
Z_{s}(f) & :=\left\{p \in M^{2} ; \operatorname{Re} \zeta_{s}(p)=0\right\} .
\end{aligned}
$$

Though $\sqrt{\zeta_{c}}$ is multi-valued on $M^{2}$, the condition $\operatorname{Im} \sqrt{\zeta_{c}}=0$ is unambiguous.

By Theorem 1.1,

- $p \in M^{2}$ is a singular point if and only if $p \in \Sigma(f)$;

- $p \in \Sigma(f)$ is a nondegenerate singular point if and only if $p \in Z_{0}(f)^{c}$;

- $p \in \Sigma(f)$ is a cuspidal edge if and only if $p \in Z_{0}(f)^{c} \cap Z_{c}(f)^{c}$;

- $p \in \Sigma(f)$ is a swallowtail if and only if $p \in Z_{0}(f)^{c} \cap Z_{c}(f) \cap Z_{s}(f)^{c}$;

- a singular point $p \in \Sigma(f)$ is neither a cuspidal edge nor a swallowtail if and only if

$$
p \in Z_{0}(f) \cup\left(Z_{c}(f) \cap Z_{s}(f)\right) .
$$


Here a superscript ${ }^{c}$ denotes the complementary set, whereas the subscript $c$ and $s$ in $Z_{c}(f)$ and $Z_{s}(f)$ were chosen because these symbols describe criteria for a singular point to be a cuspidal edge or a swallowtail, respectively.

The sets $Z_{0}(f), Z_{c}(f)$ and $Z_{s}(f)$ are the same for all the parallel fronts of $f$; that is, if $\left\{f_{t}\right\}_{t \in \mathbb{R}}$ is the family of parallel fronts of $f$, we have:

Lemma 4.6.

$$
\begin{aligned}
& \Sigma\left(f_{t}\right)=\left\{p \in M^{2} ;|\rho(p)|=e^{2 t}\right\}, \\
& Z_{0}\left(f_{t}\right)=Z_{0}(f), \quad Z_{c}\left(f_{t}\right)=Z_{c}(f), \quad Z_{s}\left(f_{t}\right)=Z_{s}(f) .
\end{aligned}
$$

Proof. By (3-8), we have the first assertion. Though the remaining parts can be proved by direct calculations, we give an alternative proof: Let $G$ and $G_{*}$ be the hyperbolic Gauss maps of $f$. Then by (3-15), we have

$$
\begin{aligned}
\xi & =\left(\frac{G_{*}^{\prime \prime}}{G_{*}^{\prime}}-\frac{G^{\prime \prime}}{G^{\prime}}+2 \frac{G^{\prime}+G_{*}^{\prime}}{G-G_{*}}\right) \hat{Q} d z^{3}, \\
\zeta_{c} & =\left(\frac{G_{*}^{\prime \prime}}{G_{*}^{\prime}}-\frac{G^{\prime \prime}}{G^{\prime}}+2 \frac{G^{\prime}+G_{*}^{\prime}}{G-G_{*}}\right)^{2} \frac{1}{\hat{Q}},
\end{aligned}
$$

and $\zeta_{s}$ is written as in (4-8). Since the hyperbolic Gauss maps and the Hopf differential are independent of the choice of parallel front $f_{t}$, we have the conclusion.

By a direct calculation using the formulas in the proof of Lemma 4.6 and (3-14), we have

$$
\zeta_{s}=\frac{\left(\sqrt{\zeta_{c}}\right)^{\prime}}{\sqrt{\hat{Q}}}
$$

with ${ }^{\prime}=d / d z$. Using this, we can prove:

Proposition 4.7. Let $f$ be a complete flat front which is not totally umbilic. Then the function $\zeta_{c}$ is constant if and only if $f$ is a covering of a front of revolution.

Proof. If $\zeta_{c}=0$, we have $d \rho=0$ on $M^{2}$. Hence $\rho$ is constant. In this case, one can conclude that $f$ is a covering of a hyperbolic cylinder, which is a surface of revolution.

On the other hand, assume $\zeta_{c}$ is a nonzero constant. By (4-3), $\hat{\theta}^{\prime} / \hat{\theta}-\hat{\omega}^{\prime} / \hat{\omega}$ can only have simple poles. Then by the definition of $\zeta_{c}$ in (4-7), the order of $Q$ is at least -2 . Thus, by Fact 4.2 , all ends must be regular.

By (4-9), $\zeta_{s}=0$ holds. Then by (4-8), we have $\{G, z\}=\left\{G_{*}, z\right\}$ with respect to any complex coordinate $z$. Then

$$
G_{*}=b \star G=\frac{b_{11} G+b_{12}}{b_{21} G+b_{22}}, \quad b=\left(\begin{array}{ll}
b_{11} & b_{12} \\
b_{21} & b_{22}
\end{array}\right) \in \operatorname{SL}(2, \mathbb{C}),
$$


where $\star$ denotes the Möbius transformation. Here, the group $\operatorname{SL}(2, \mathbb{C})$ acts isometrically on $H^{3}$ as

$$
H^{3} \ni x \mapsto a x a^{*} \in H^{3} \quad a \in \operatorname{SL}(2, \mathbb{C}),
$$

where we consider $H^{3}$ as in (1-2). Under the isometry (4-11), the hyperbolic Gauss maps transform as $\left(G, G_{*}\right) \mapsto\left(a \star G, a \star G_{*}\right)$. Hence we may assume $b$ in (4-10) is a Jordan normal form.

When $b$ is diagonal, we have $G_{*}=\mu G$, where $\mu$ is constant. Here, since $f$ is a flat front, $G$ and $G_{*}$ have no common branch points; see [Kokubu et al. 2004]. Thus $G$ has no branch point, and then we can take $z=G$ as a local coordinate. Hence $f$ is locally congruent to a front of revolution (see Example 6.2 on page 344). Thus we have the conclusion. If $b$ is not diagonal, the eigenvalue of $b$ is \pm 1 , which is a double root. Then we have $G_{*}=G-1$. Since the ends of $f$ are the points where $G=G_{*}$ ([Kokubu et al. 2004, Lemma 4.10]), the ends are common poles of $G$ and $G_{*}$. In this case, by (3-14) we have $Q=-d G d G_{*}=-d G^{2}$. Then the ord $\operatorname{or}_{p} Q$ at a pole $p$ of $G$ is less than or equal to -4 , which contradicts the fact that all ends are regular.

Proof of Theorem 1.2. Let

$$
f: M^{2}=\bar{M}^{2} \backslash\left\{p_{1}, \ldots, p_{N}\right\} \rightarrow H^{3}
$$

be a complete flat front which is not totally umbilic, and $\left\{f_{t}\right\}$ its parallel family. For simplicity, we write

$\Sigma_{t}:=\Sigma\left(f_{t}\right), Z_{0}:=Z_{0}\left(f_{t}\right)=Z_{0}(f), Z_{c}:=Z_{c}\left(f_{t}\right)=Z_{c}(f), Z_{s}:=Z_{s}\left(f_{t}\right)=Z_{s}(f)$.

A point $p \in M^{2}$ is a singular point of $f_{t}$ that is neither a cuspidal edge nor a swallowtail if and only if

$$
p \in \Sigma_{t} \cap\left(Z_{0} \cup\left(Z_{c} \cap Z_{s}\right)\right) .
$$

Then by Lemma 4.6, $f_{t}$ admits such a singular point if and only if

$$
\left\{|\rho(p)| ; p \in Z_{0} \cup\left(Z_{c} \cap Z_{s}\right)\right\} \ni e^{2 t} .
$$

Since $\xi$ in (4-7) is a meromorphic 3-differential on the compact Riemann surface $\bar{M}^{2}$ and $Z_{0}$ is the set of zeroes of $\xi, Z_{0}$ is a finite set of points. We now take for granted the following result, to be proved shortly:

Proposition 4.8. Let $f$ be a complete flat front such that $\zeta_{c}$ defined in (4-7) is not constant. Then $\left\{|\rho(p)| ; p \in Z_{c} \cap Z_{s}\right\} \subset \mathbb{R}_{+}$is a finite set.

Assume a complete flat front $f$ is a front of revolution. Such a flat front is a horosphere, a finite cover of a hyperbolic cylinder, a snowman, or an hourglass (see Example 6.2). Among these, the horospheres and hyperbolic cylinders do not have 
singular points, and all singularities of the snowman are cuspidal edges. Since we assumed $f$ is not a cover of an hourglass, we have the conclusion for the case of fronts of revolution.

Next, we assume $f$ is not a front of revolution. Then by Proposition 4.7, $\zeta_{c}$ is nonconstant. Hence by Proposition 4.8, $\left\{|\rho(p)| ; p \in Z_{c} \cap Z_{s}\right\}$ is a finite set. On the other hand, the parallel front $f_{t}$ admits a singular point which is neither a cuspidal edge nor a swallowtail if and only if (4-13) holds. Hence we have the conclusion.

Lemma 4.9. Let $f$ be a complete flat front as in (4-12) with nonconstant $\zeta_{c}$. Assume $Z_{c} \cap Z_{s}$ accumulates at a point $p \in \bar{M}^{2}$. Then

(1) $p$ is a nonumbilic point in $M^{2}$ or an irregular cylindrical end, and

(2) there exists a neighborhood $U$ of $p$ such that the number of connected components of

$$
(U \backslash\{p\}) \cap\left(Z_{c} \cap Z_{s}\right)
$$

is finite, and each connected component is a level set of $|\rho|$.

Proof of Proposition 4.8 assuming Lemma 4.9. Suppose

$$
\#\left\{|\rho(p)| ; p \in Z_{c} \cap Z_{s}\right\}=+\infty .
$$

Then there exists an infinite sequence $\left\{z_{n}\right\} \subset Z_{c} \cap Z_{s}$ such that $\left|\rho\left(z_{n}\right)\right|(n=1,2, \ldots)$ are mutually distinct. Since $\bar{M}^{2}$ is compact, we can take a subsequence of $\left\{z_{n}\right\}$

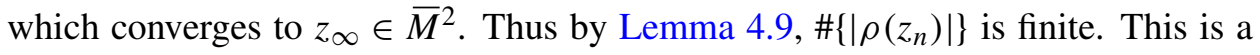
contradiction because the $\left|\rho\left(z_{n}\right)\right|(n=1,2, \ldots)$ are mutually distinct.

Proof of the Lemma 4.9(1). Let $p$ be an accumulation point of $Z_{c} \cap Z_{s}$, and take a sequence $\left\{p_{n}\right\}$ consisting of mutually distinct points in $Z_{c} \cap Z_{s}$ such that $p_{n} \rightarrow p$ as $n \rightarrow \infty$. We show the first assertion of the lemma by way of contradiction: We assume

- $p \in M^{2}$ is an umbilic point, or

- $p \in \bar{M}^{2}$ is an end which is not an irregular cylindrical end,

and set

$$
\mu=\operatorname{ord}_{p} \omega, \quad \mu_{*}=\operatorname{ord}_{p} \theta \quad \text { and } \quad k=\operatorname{ord}_{p} Q=\mu+\mu_{*} \in \mathbb{Z} .
$$

If $p$ is an umbilic point, $\mu \neq \mu_{*}$ holds because of (4-5). If $p$ is an end, $\mu=\mu_{*}$ holds when $p$ is cylindrical. We consider the two cases separately.

Case 1: $\mu \neq \mu_{*}$, that is, $p$ is an umbilic point or a noncylindrical end. If we take a complex coordinate $z$ around $p$ such that $z(p)=0$, we can write

$$
\frac{\hat{\theta}^{\prime}}{\hat{\theta}}-\frac{\hat{\omega}^{\prime}}{\hat{\omega}}=\frac{a}{z}(1+O(z)) \quad\left(a:=\mu_{*}-\mu\right),
$$


where $O(z)$ denotes a higher-order term. On the other hand, the Hopf differential $Q$ is written as

$$
Q=z^{k}\left(q_{0}+O(z)\right) d z^{2} \quad\left(q_{0} \neq 0\right) .
$$

Thus, it follows from (4-7) that

$$
\sqrt{\zeta_{c}}=z^{-(k+2) / 2}\left(\frac{a}{\sqrt{q_{0}}}+O(z)\right) .
$$

We assume $k \neq-2$. Then by (4-14), (4-15) and (4-9), we have

$$
\zeta_{s}=z^{-k-2}\left(\frac{a}{q_{0}}+O(z)\right) .
$$

Let $z_{n}=z\left(p_{n}\right)$. Then $z_{n}$ tends to the origin as $n \rightarrow \infty$. Since $p_{n} \in Z_{c} \cap Z_{s}$, we have $\operatorname{Im} \sqrt{\zeta_{c}\left(z_{n}\right)}=\operatorname{Re} \zeta_{s}\left(z_{n}\right)=0$. Since $a \in \mathbb{R}$, there exist sequences $\left\{\varepsilon_{n}\right\}$ and $\left\{\varepsilon_{n}^{\prime}\right\}$ of real numbers such that

$$
\begin{aligned}
& 0 \equiv \arg \sqrt{\zeta_{c}\left(z_{n}\right)}=-\left(\frac{k}{2}+1\right) \arg z_{n}-\frac{1}{2} \arg q_{0}+\varepsilon_{n} \quad(\bmod \pi), \\
& \frac{\pi}{2} \equiv \arg \zeta_{s}\left(z_{n}\right)=-(k+2) \arg z_{n}-\arg q_{0}+\varepsilon_{n}^{\prime} \quad(\bmod \pi),
\end{aligned}
$$

and $\varepsilon_{n}, \varepsilon_{n}^{\prime} \rightarrow 0$ as $n \rightarrow \infty$. But this implies

$$
-\frac{\pi}{2} \equiv 2 \arg \sqrt{\zeta_{c}\left(z_{n}\right)}-\arg \zeta_{s}\left(z_{n}\right) \equiv 2 \varepsilon_{n}-\varepsilon_{n}^{\prime} \quad(\bmod \pi),
$$

giving a contradiction. Then the case $k \neq-2$ is impossible.

Assume $k=-2$. In this case, $(4-15)$ is written as $\sqrt{\zeta_{c}}=a q_{0}^{-1 / 2}+O(z)$. Then by the assumption that $\zeta_{c}$ is nonconstant, there exists a positive integer $l$ such that

$$
\sqrt{\zeta_{c}}=\frac{a}{\sqrt{q_{0}}}+b z^{l}+O\left(z^{l+1}\right) \quad(b \neq 0) .
$$

In this case, by (4-9), we have

$$
\zeta_{s}=z^{l}\left(\frac{l b}{\sqrt{q_{0}}}+O(z)\right)
$$

Here $\operatorname{Im} \sqrt{\zeta_{c}\left(z_{n}\right)}=0$ holds on a sequence $\left\{z_{n}=z\left(p_{n}\right)\right\}$ such that $z_{n} \rightarrow 0$ as $n \rightarrow \infty$, and $a \in \mathbb{R}$. Hence (4-16) implies that $\sqrt{q_{0}} \in \mathbb{R}$. Thus, we have

$$
\begin{aligned}
0 & \equiv \arg \sqrt{\zeta_{c}\left(z_{n}\right)}=\arg \left(b z_{n}^{l}+O\left(z^{l+1}\right)\right)=l \arg z_{n}+\arg b+\varepsilon_{n} & & (\bmod \pi), \\
\frac{\pi}{2} \equiv \arg \zeta_{s}\left(z_{n}\right) & =l \arg z_{n}+\arg b+\varepsilon_{n}^{\prime}, & & (\bmod \pi),
\end{aligned}
$$

where $\varepsilon_{n}, \varepsilon_{n}^{\prime} \rightarrow 0$ as $n \rightarrow \infty$. Again, the two displayed equations contradict each other. 
Case 2: $\mu=\mu_{*}$, that is, $p$ is a cylindrical end. In this case, $p$ is a regular end because of our assumption. By Fact 4.2 and (4-4), we have $\mu=\mu_{*}=-1$. Taking a complex coordinate $z$ such that $z(p)=0$, we can write $\hat{Q}=z^{-2}\left(q_{0}+O(z)\right)$. Denote by $d \in \mathbb{Z}_{+} \cup\{0\}$ the branch order of $G$ at $z=0$. (For example, if $G=a+z^{d+1}$, the branch order of $G$ at $z=0$ is $d$.) Since $\mu=-1$, (3-16) implies that

$$
q_{0}=\frac{1}{4}(d+1)^{2}>0 .
$$

Since $\mu=\mu_{*}=-1$, we have $\left(\hat{\theta}^{\prime} / \hat{\theta}\right)-\left(\hat{\omega}^{\prime} / \hat{\omega}\right)=O(1)$. Hence $\sqrt{\zeta_{c}(z)}=O(z)$. Thus, we can write

$$
\sqrt{\zeta_{c}}=b z^{l}+O\left(z^{l+1}\right), \quad \hat{Q}=\frac{1}{z^{2}}\left(\frac{1}{4}(d+1)^{2}+O(z)\right),
$$

where $l \geq 1$ is an integer and $b \neq 0$. Thus,

$$
\zeta_{s}=\frac{l b}{\sqrt{q_{0}}} z^{l}(1+O(z))=\frac{l b}{d+1} z^{l}(1+O(z)) .
$$

As in Case 1 , we set $z_{n}=z\left(p_{n}\right)$. Then we have

$$
\arg b+l \arg z_{n}+\varepsilon_{n} \equiv 0, \quad \arg b+l \arg z_{n}+\varepsilon_{n}^{\prime} \equiv \frac{\pi}{2} \quad(\bmod \pi),
$$

where $\varepsilon_{n}, \varepsilon_{n}^{\prime} \rightarrow 0$. This is impossible.

Hence in any case, $Z_{c} \cap Z_{s}$ does not accumulate at 0 .

Proof of Lemma 4.9(2). We consider two cases.

Case 1: Suppose that $Z_{c} \cap Z_{s}$ accumulates at a nonumbilic point $p \in M^{2}$, i.e., $Q(p) \neq 0$.

Take a complex coordinate $z$ around $p$ with $z(p)=0$. Since $\hat{Q}(0) \neq 0$, there exists a holomorphic function $\varphi(z)$ defined on a neighborhood of the origin such that

$$
(\varphi(z))^{2}=\hat{Q}(z) ; \quad \text { that is, } \quad \varphi(z)=\sqrt{\hat{Q}(z)}, \quad \text { and } \quad \varphi(0) \neq 0 .
$$

On the other hand, both $\hat{\theta}$ and $\hat{\omega}$ have neither a zero nor a pole at $z=0$, so by (4-7), $\sqrt{\zeta_{c}}$ is a holomorphic function near $z=0$. Since $\zeta_{c}$ is not a constant, there exists a positive integer $l$ such that $\sqrt{\zeta_{c}}=a+b z^{l}+O\left(z^{l+1}\right)$, where $b \neq 0$. Then by the Weierstrass preparation theorem, we can choose a coordinate $z$ such that

$$
\sqrt{\zeta_{c}(z)}=a+z^{l}, \quad \varphi(z)=\sqrt{\hat{Q}(z)}=\varphi_{0}+O(z) \quad\left(l \in \mathbb{Z}_{+}, \varphi_{0} \in \mathbb{C} \backslash\{0\}\right) .
$$

Moreover, replacing $\varphi_{0} z$ by $z$, we can set

$$
\text { (4-17) } \sqrt{\zeta_{c}(z)}=a+b z^{l}, \quad \varphi(z)=1+O(z) \quad\left(l \in \mathbb{Z}_{+}, b=\varphi_{0}^{-l} \in \mathbb{C} \backslash\{0\}\right) .
$$


Here, since $Z_{c}$ accumulates at $0, a$ in (4-17) must be real, and then

$$
\operatorname{Im} \sqrt{\zeta_{c}}=\operatorname{Im}\left(b z^{l}\right)
$$

On the other hand, by (4-9),

$$
\zeta_{s}=\frac{l b z^{l-1}}{\varphi(z)}=l b z^{l-1}(1+O(z)) .
$$

We identify a neighborhood of $p$ with a neighborhood of the origin of the $z$-plane. Since $Z_{c} \cap Z_{s}$ accumulates to the origin, we can take a sequence $\left\{z_{n}\right\} \subset Z_{c} \cap Z_{s}$ such that $z_{n} \rightarrow 0$ as $n \rightarrow \infty$. Then by (4-18) and (4-19), there exists a sequence $\left\{\varepsilon_{n}\right\} \subset \mathbb{R}$ such that $\varepsilon_{n} \rightarrow 0$ and

$$
\begin{array}{rlrl}
\arg b+l \arg z_{n} & \equiv 0 & & (\bmod \pi), \\
\arg b+(l-1) \arg z_{n}+\varepsilon_{n} \equiv \frac{\pi}{2} & (\bmod \pi),
\end{array}
$$

hold. Subtracting (4-21) from (4-20), we have

$$
\arg z_{n}-\varepsilon_{n} \equiv \frac{\pi}{2} \quad(\bmod \pi) .
$$

On the other hand, subtracting (4-20) multiplied by $l-1$ from (4-21) multiplied by $l$, we have

$$
\arg b \equiv l \frac{\pi}{2}-l \varepsilon_{n} \quad(\bmod \pi) .
$$

Here, since $\varepsilon_{n} \rightarrow 0$, we deduce that

$$
\arg b \equiv l \frac{\pi}{2} \quad(\bmod \pi) \quad \text { and } \quad \varepsilon_{n}=0 .
$$

Substituting these into (4-22), we have $\arg z_{n} \equiv \pi / 2(\bmod \pi)$; that is, $z_{n} \in \sqrt{-1} \mathbb{R}$. Since $\sqrt{\zeta_{c}\left(z_{n}\right)} \in \mathbb{R}$ for $n=1,2, \ldots$, the imaginary part of $\sqrt{\zeta_{c}(z)}$ vanishes identically on $\sqrt{-1} \mathbb{R}$, namely,

$$
\sqrt{\zeta_{c}(z)} \in \mathbb{R} \quad(\text { if } z \in \sqrt{-1} \mathbb{R}) .
$$

Similarly, one can prove that

$$
\zeta_{s}(z) \in \sqrt{-1} \mathbb{R} \quad(\text { if } z \in \sqrt{-1} \mathbb{R}) .
$$

Thus, on a neighborhood of the origin, $Z_{c} \cap Z_{s}$ is the imaginary axis in the $z$-plane. 
Next, we prove that the imaginary axis is a level set of $|\rho|$. By (4-17), (4-19) and (4-23),

$$
\begin{aligned}
\frac{\pi}{2} \equiv \arg \zeta_{s}(z) & =\arg \left(l b z^{l-1} \frac{1}{\varphi}\right)=\arg \left(b z^{l}\right)-\arg z \varphi(z) \\
& =\arg \sqrt{\zeta_{c}(z)}-\arg z-\arg \varphi(z) \\
& =-\arg z-\arg \varphi(z)=-\frac{\pi}{2}-\arg \varphi(z) \quad(\bmod \pi)
\end{aligned}
$$

holds on the imaginary axis. Thus

$$
\varphi(z) \in \mathbb{R} \quad \text { (if } z \in \sqrt{-1} \mathbb{R}) .
$$

As seen in (3-11) in Section 3, the tangent vector field of a level set of $|\rho|$ is represented as

At the same time,

$$
\sqrt{-1} \overline{\left(\frac{\hat{\theta}^{\prime}}{\hat{\theta}}-\frac{\hat{\omega}^{\prime}}{\hat{\omega}}\right)} .
$$

$$
\sqrt{\zeta_{c}(z)}=\frac{1}{\varphi(z)}\left(\frac{\hat{\theta}^{\prime}}{\hat{\theta}}-\frac{\hat{\omega}^{\prime}}{\hat{\omega}}\right) \in \mathbb{R} \quad \text { and } \quad \varphi(z) \in \mathbb{R} \quad(\text { if } z \in \sqrt{-1} \mathbb{R}) .
$$

Without loss of generality, $d \rho \neq 0$ holds on $U \backslash\{0\}$, where $U$ is a neighborhood of the origin, because a zero of $d \rho$ is isolated in $\bar{M}^{2}$. Then the tangent vector of the level set of $|\rho|$ at a point on the imaginary axis is parallel to the imaginary axis. Hence the level set passing through a point of the imaginary axis is the imaginary axis. That is, $Z_{c} \cap Z_{s}$ coincides with the imaginary axis, which is a level set of $|\rho|$. Case 2: Suppose now that $Z_{c} \cap Z_{s}$ accumulates at an irregular cylindrical end $p$. Let $z$ be a complex coordinate with $z(p)=0$. By irregularity, $\operatorname{ord}_{p} Q \leq-3$. Without loss of generality, we may assume $\operatorname{ord}_{p} Q$ is an even number. In fact, if we set $z=w^{2}$, that is, we take the double cover of a neighborhood of $p$, the order of $Q$ at the origin with respect to the coordinate $w$ will be an even number.

Hence, we assume

$$
\operatorname{ord}_{p} Q=-2 k, \quad \operatorname{ord}_{p} \omega=\operatorname{ord}_{p} \theta=-k,
$$

where $k \geq 2$ is an integer. The second equality holds because $p$ is a cylindrical end.

Since $Q$ has even order at the origin, $\sqrt{\hat{Q}}$ is a meromorphic function on a neighborhood of 0 . More precisely, we can write

$$
\varphi:=\sqrt{\hat{Q}}=\frac{1}{z^{k}}\left(\varphi_{0}+O(z)\right) \quad\left(\varphi_{0} \in \mathbb{C} \backslash\{0\}\right) .
$$

Since $\operatorname{ord}_{p} \omega=\operatorname{ord}_{p} \theta$, (4-7) implies that $\sqrt{\zeta_{c}(z)}=O\left(z^{k}\right)$, that is, there exists an integer $l(l \geq k)$ such that $\sqrt{\zeta_{c}(z)}=a z^{l}+O\left(z^{l+1}\right)(a \in \mathbb{C} \backslash\{0\})$. Then, by the 
Weierstrass preparation theorem, we can choose a coordinate $z$ such that

$$
\sqrt{\zeta_{c}(z)}=z^{l}, \quad \varphi=\frac{1}{z^{k}}(b+O(z)) \quad(b \in \mathbb{C} \backslash\{0\}) .
$$

Then, by (4-9), $\zeta_{s}$ is written as

$$
\zeta_{s}(z)=l z^{l+k-1}\left(\frac{1}{b}+O(z)\right) .
$$

Since $\left\{z_{n}\right\} \subset Z_{c} \cap Z_{s}$ is a sequence with $z_{n} \rightarrow 0$, we have

$$
\begin{aligned}
l \arg z_{n} & \equiv 0 & & (\bmod \pi), \\
-\arg b+(l+k-1) \arg z_{n}+\varepsilon_{n} & \equiv \frac{\pi}{2} & & (\bmod \pi),
\end{aligned}
$$

where $\varepsilon_{n} \rightarrow 0$. Subtracting (4-26) from (4-25), and (4-25) multiplied by $l+k-1$ from (4-26) multiplied by $l$, we have

$$
-\arg b+(k-1) \arg z_{n}+\varepsilon_{n} \equiv \frac{\pi}{2}, \quad-l \arg b+l \varepsilon_{n} \equiv l \frac{\pi}{2} \quad(\bmod \pi) .
$$

Since $\varepsilon_{n} \rightarrow 0$, this yields $-l \arg b \equiv l \pi / 2(\bmod \pi)$, and then $\varepsilon_{n}=0$ for sufficiently large $n$. Thus, we have

$$
(k-1) \arg z_{n} \equiv \frac{\pi}{2}+\arg b \quad(\bmod \pi) .
$$

Let

$$
\mathscr{L}_{j}:=\left\{z \in U ; \arg z \equiv \frac{1+2 j}{2(k-1)} \pi+\frac{\arg b}{k-1} \quad(\bmod \pi)\right\} \quad(j=0, \ldots, k-1) .
$$

Then $\left\{\mathscr{L}_{j}\right\}$ is a set consisting of a finite number of lines in the $z$-plane through the origin, and by (4-27), each $z_{n}$ lies on some $\mathscr{L}_{j}$. Hence there exists a subset $J \subset\{0,1,2, \ldots, k-1\}$ such that each $\mathscr{L}_{j}(j \in J)$ contains infinitely many elements of $\left\{z_{n}\right\}$. We fix $j \in J$. Then we can take a subsequence $\left\{z_{m}\right\}$ of $\left\{z_{n}\right\}$ such that $z_{m} \in \mathscr{L}_{j} \cap Z_{c} \cap Z_{s}$ and $z_{m} \rightarrow 0$. Since $\zeta_{c}\left(z_{m}\right) \in \mathbb{R}$ and $\zeta_{s}\left(z_{m}\right) \in \sqrt{-1} \mathbb{R}$, we have

$$
\sqrt{\zeta_{c}(z)} \in \mathbb{R}, \quad \zeta_{s}(z) \in \sqrt{-1} \mathbb{R} \quad \text { (if } z \in \mathscr{L}_{j} \text { ). }
$$

This shows that, on a neighborhood of the origin, $Z_{c} \cap Z_{s}$ coincides with the set of lines $\bigcup_{j \in J} \mathscr{L}_{j}$.

Next, we show that $\mathscr{L}_{j}(j \in J)$ is a level set of $|\rho|$ for each $j$. By (4-24) and (4-28),

$$
\begin{aligned}
\frac{\pi}{2} \equiv \arg \zeta_{s}(z) & =\arg \sqrt{\zeta_{c}(z)}-\arg z-\arg \varphi \\
& =-\frac{1+2 j}{2(k-1)} \pi-\frac{\arg b}{k-1}-\arg \varphi \quad(\bmod \pi)
\end{aligned}
$$


holds on $\mathscr{L}_{j}$. Hence

$$
\arg \varphi \equiv \frac{\pi}{2}-\frac{1+2 j}{2(k-1)} \pi-\frac{\arg b}{k-1} \quad(\bmod \pi) \quad\left(\text { if } z \in \mathscr{L}_{j}\right) .
$$

At any point in $\mathscr{L}_{j}$, the argument of the tangent vector of the level set of $|\rho|$ is

$$
\begin{aligned}
\arg \sqrt{-1} \overline{\left(\frac{\hat{\theta}^{\prime}}{\hat{\theta}}-\frac{\hat{\omega}^{\prime}}{\hat{\omega}}\right)} & \equiv \arg \left(\sqrt{-1} \overline{\left(\sqrt{\zeta_{c}} \varphi\right)}\right) \equiv \frac{\pi}{2}-\arg \varphi-\arg \sqrt{\zeta_{c}} \\
& \equiv \frac{\pi}{2}-\arg \varphi=\frac{1+2 j}{2(k-1)} \pi+\frac{\arg b}{k-1} \quad(\bmod \pi),
\end{aligned}
$$

and then, the tangent vector is proportional to the line $\mathscr{L}_{j}$. Hence each $\mathscr{L}_{j}(j \in J)$ is a level set of $|\rho|$. Thus, we have the conclusion.

Remark 4.10. Let $p \in \bar{M}^{2}$ be an accumulation point of $Z_{c} \cap Z_{s}$. Then by Lemma 4.9(2), $Z_{c} \cap Z_{s}$ is a level set of $|\rho|$ in a neighborhood of $p$; that is, by taking a suitable parallel front, we may assume that a component of $Z_{c} \cap Z_{s}$ is a part of the singular set. Since the null direction and the singular direction coincide at each point in $Z_{c} \cap Z_{s}$, the image of such a singular set is a single point in $H^{3}$. If the point $p$ is not an end, such a singularity seems to be a so-called cone-like singularity, see, for example, the hourglass in Example 6.2. Another example is as follows: Set

$$
\omega=\exp \left(z+\frac{1}{3} z^{3}\right) d z, \quad \theta=\exp \left(-z-\frac{1}{3} z^{3}\right) d z
$$

on $\mathbb{C}$. Then by solving (3-2), we have a flat front $f: \mathbb{C} \rightarrow H^{3}$. The singular set of $f$ contains the imaginary axis, which coincides with $Z_{c} \cap Z_{s}$ (see figure). However, this example is not complete because the canonical forms have an essential singularity at $z=\infty$.

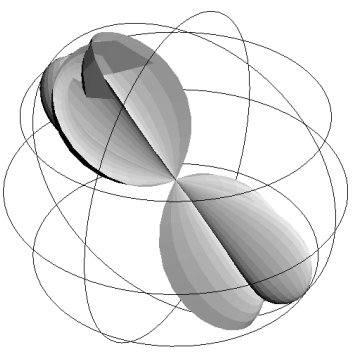

\section{Caustics of flat fronts}

Roitman [2003] has investigated the caustic of (the parallel family of) a flat front, considered as the locus of singular points of the fronts in the parallel family. In this section, we discuss caustics of flat fronts from our point of view.

Let $U \subset \mathbb{C}$ be a simply connected domain and $f: U \rightarrow H^{3}$ a flat front without umbilic points. We denote by $\omega$ and $\theta$ the canonical forms of $f$, and $\rho:=\theta / \omega$, as 
in the previous section. Since $f$ has no umbilic points, $\rho$ does not take values 0 and $\infty$.

For a point $z \in U$, we denote by $\kappa_{1}$ and $\kappa_{2}$ the principal curvatures of $f$ at $z$. Then by (3-4),

$$
\kappa_{1}=\frac{|\rho|+1}{|\rho|-1} \quad \text { and } \quad \kappa_{2}=\frac{|\rho|-1}{|\rho|+1} .
$$

Since $\rho \neq 0, \infty$, we have $\left|\kappa_{1}\right|>1$. Then there exists a real number $r_{1}$ such that coth $r_{1}=\kappa_{1}$, called the radius of curvature. By (5-1),

$$
e^{2 r_{1}}=|\rho| \text {. }
$$

The caustic $C_{f}$ of $f$ is defined as

$$
C_{f}: U \ni z \mapsto \cosh r_{1}(z) f(z)+\sinh r_{1}(z) v(z) \in H^{3} \subset \boldsymbol{L}^{4},
$$

where $\boldsymbol{L}^{4}$ is the Minkowski 4-space and $v$ is the unit normal vector of $f$. In other words, $C_{f}$ is the locus of the centers of the principal curvature $\kappa_{1}$ of $f$.

Let $E_{f}: U \rightarrow \operatorname{SL}(2, \mathbb{C})$ be the holomorphic lift of the front $f$. Then $f$ and the unit normal vector $v$ are given by

$$
f=E_{f} E_{f}^{*}, \quad v=E_{f}\left(\begin{array}{rr}
1 & 0 \\
0 & -1
\end{array}\right) E_{f}^{*} .
$$

Thus, the caustic of $f$ is

$$
\begin{aligned}
C_{f} & =E_{f}\left(\cosh r_{1}\left(\begin{array}{ll}
1 & 0 \\
0 & 1
\end{array}\right)+\sinh r_{1}\left(\begin{array}{rr}
1 & 0 \\
0 & -1
\end{array}\right)\right) E_{f}^{*} \\
& =E_{f}\left(\begin{array}{cc}
e^{r_{1}} & 0 \\
0 & e^{-r_{1}}
\end{array}\right) E_{f}^{*}=E_{f}\left(\begin{array}{cc}
|\rho|^{1 / 2} & 0 \\
0 & |\rho|^{-1 / 2}
\end{array}\right) E_{f}^{*} .
\end{aligned}
$$

Hence if we set

$$
E_{c}=E_{f}\left(\begin{array}{cc}
\rho^{1 / 4} & 0 \\
0 & \rho^{-1 / 4}
\end{array}\right) P, \quad P=\frac{1}{\sqrt{2}}\left(\begin{array}{cc}
1 & \sqrt{-1} \\
\sqrt{-1} & 1
\end{array}\right) \in \mathrm{SU}(2),
$$

we have

$$
C_{f}=E_{c} E_{c}^{*}, \quad \text { and } \quad E_{c}^{-1} d E_{c}=\left(\begin{array}{cc}
0 & \theta_{c} \\
\omega_{c} & 0
\end{array}\right)
$$

where

$(5-5)$

$$
\omega_{c}=\sqrt{\hat{\omega} \hat{\theta}} d z-\sqrt{-1} \frac{d \rho}{4 \rho}, \quad \theta_{c}=\sqrt{\hat{\omega} \hat{\theta}} d z+\sqrt{-1} \frac{d \rho}{4 \rho} \quad(\omega=\hat{\omega} d z, \theta=\hat{\theta} d z)
$$

Since $U$ contains no umbilic points of $f$, both $\omega$ and $\theta$ have no zeroes. Thus $\omega_{c}$ and $\theta_{c}$ have no common zero, which implies that: 
Theorem 5.1 [Roitman 2003]. The caustic $C_{f}=E_{c} E_{c}^{*}: U \rightarrow H^{3}$ of a flat front $f: U \rightarrow H^{3}$ without umbilic points is a flat front with canonical forms $\omega_{c}$ and $\theta_{c}$ as in (5-5). Moreover, we have

$$
E_{c}=\frac{(-1)^{1 / 4} \alpha^{-1 / 4}}{\sqrt{2} \sqrt{G-G_{*}}}\left(\begin{array}{cc}
G+\sqrt{\alpha} G_{*} & \sqrt{-1}\left(G-\sqrt{\alpha} G_{*}\right) \\
1+\sqrt{\alpha} & \sqrt{-1}(1-\sqrt{\alpha})
\end{array}\right) \quad\left(\alpha=\frac{d G}{d G_{*}}\right),
$$

where $G$ and $G_{*}$ are the hyperbolic Gauss maps of $f$. In particular, the hyperbolic Gauss maps $\left(G_{c}, G_{c, *}\right)$ of $C_{f}$ are given by

$$
G_{c}=\frac{G+\sqrt{\alpha} G_{*}}{1+\sqrt{\alpha}}, \quad G_{c, *}=\frac{G-\sqrt{\alpha} G_{*}}{1-\sqrt{\alpha}} .
$$

If $z$ is a singular point of $f$, we have $r_{1}(z)=0$ because $|\rho(z)|=1$. Therefore, the caustic of a parallel family $\left\{f_{t}\right\}$ of flat fronts is the locus of singular points of the fronts $f_{t}$ for $t \in \mathbb{R}$.

Since the parallel family has a common caustic, the sets $Z_{0}, Z_{c}$ and $Z_{s}$ (page 331) can be considered as well-defined on the caustic. In particular:

Proposition 5.2. Let $f: U \rightarrow H^{3}$ be a flat front without umbilic points, and with caustic $C_{f}$, where $U \subset \mathbb{C}$ is a simply connected domain. Then

(1) A point $p \in U$ is a singular point of the caustic $C_{f}$ if and only if $p \in Z_{c}(f)$.

(2) A point $p \in Z_{c}(f)$ is a nondegenerate singular point of the caustic if and only if $S(G)-S\left(G_{*}\right) \neq 0$ holds at $p$, where $G$ and $G_{*}$ are the hyperbolic Gauss maps of $f$.

(3) A point $p \in Z_{c}(f)$ where $S(G)-S\left(G_{*}\right) \neq 0$ is a cuspidal edge of the caustic if and only if $p \notin Z_{s}(f)$.

In other words, the locus of the cuspidal edges of $\left\{f_{t}\right\}_{t \in \mathbb{R}}$ is the set of regular points of the caustic. Furthermore, the locus of the swallowtails of $\left\{f_{t}\right\}$ is the set of cuspidal edges on the caustic, except the points at which $S(G)-S\left(G_{*}\right)=0$.

Proof of Proposition 5.2. A point $p \in U$ is a singular point of $C_{f}$ if and only if $\left|\omega_{c}\right|^{2}=\left|\theta_{c}\right|^{2}$. By (5-5), this is equivalent to

$$
0=\operatorname{Im} \overline{\sqrt{\hat{\omega} \hat{\theta}}} \frac{\rho^{\prime}}{\rho}=\operatorname{Im}\left(|\hat{\omega} \hat{\theta}| \frac{1}{\sqrt{\hat{\omega} \hat{\theta}}}\left(\frac{\hat{\theta}^{\prime}}{\hat{\theta}}-\frac{\hat{\omega}^{\prime}}{\hat{\omega}}\right)\right)=|\hat{\omega} \hat{\theta}| \operatorname{Im} \sqrt{\zeta_{c}} .
$$

Hence the first assertion holds.

In this case, $p$ is a degenerate singular point of $C_{f}$ if and only if

$$
0=\hat{\theta}_{c}^{\prime} \hat{\omega}_{c}-\hat{\omega}_{c}^{\prime} \hat{\theta}_{c}=\sqrt{\hat{\omega} \hat{\theta}}(s(\hat{\theta})-s(\hat{\omega})) .
$$

Then by (3-16), we have the second assertion. 
Finally, if $p$ is a nondegenerate singular point of $C_{f}, p$ is a cuspidal edge if and only if

$$
\operatorname{Im} \frac{1}{\sqrt{\hat{\omega}_{c} \hat{\theta}_{c}}}\left(\frac{\hat{\theta}_{c}^{\prime}}{\hat{\theta}_{c}}-\frac{\hat{\omega}_{c}^{\prime}}{\hat{\omega}_{c}}\right) \neq 0 .
$$

Here, by direct calculation,

$$
\frac{1}{\sqrt{\hat{\omega}_{c} \hat{\theta}_{c}}}\left(\frac{\hat{\theta}_{c}^{\prime}}{\hat{\theta}_{c}}-\frac{\hat{\omega}_{c}^{\prime}}{\hat{\omega}_{c}}\right)=\frac{1}{{\sqrt{\hat{\omega}_{c} \hat{\theta}_{c}^{3}}}^{3}} \frac{\sqrt{-1}}{4} \sqrt{\hat{\omega} \hat{\theta}^{3}}(s(\hat{\theta})-s(\hat{\omega}))=\sqrt{-1} \frac{\zeta_{s}(z)}{16+\zeta_{c}(z)} .
$$

Since $\zeta_{c}(z)$ is a positive real number if $z \in Z_{c},(5-6)$ holds if and only if $\zeta_{s}(z)$ does not lie in $\sqrt{-1} \mathbb{R}$.

\section{Examples}

Here we give examples that reaffirm the properties of singularities in Theorems 1.1 and 1.2. We make examples of flat fronts by choosing hyperbolic Gauss maps $G$ and $G_{*}$ as follows: Let $G$ and $G_{*}$ be meromorphic functions on a compact Riemann surface $\bar{M}^{2}$ such that $G$ is not identically equal to $G_{*}$, and let

$$
\left\{p_{1}, \ldots, p_{N}\right\}=\left\{p \in \bar{M}^{2} ; G(p)=G_{*}(p)\right\} \quad \text { and } \quad M^{2}=\bar{M}^{2} \backslash\left\{p_{1}, \ldots, p_{N}\right\} .
$$

If the period condition

$$
\oint_{\gamma} \frac{d G}{G-G_{*}} \in \sqrt{-1} \mathbb{R}
$$

holds for any loop $\gamma$ on $M^{2}$, we have the parallel family of a complete flat front

$$
f_{t}: M^{2}=\bar{M}^{2} \backslash\left\{p_{1}, \ldots, p_{N}\right\} \rightarrow H^{3}
$$

by substituting $G$ and $G_{*}$ in the representation formula (3-13) in Remark 3.3 with $c=e^{-t / 2}$. Moreover, by (3-14), we have

$$
\rho=-\frac{\Delta^{4}}{\left(G-G_{*}\right)^{2}} \frac{d G_{*}}{d G} .
$$

For details, see [Kokubu et al. 2004].

Example 6.1 (Cylinders). Let $G=z$ and $G_{*}=$ $1 / z$ on $\bar{M}^{2}=\mathbb{C} \cup\{\infty\}$. Then $\Delta=e^{-t / 2} \sqrt{z^{2}-1}$ and $\rho(z)=e^{-2 t}$. So $|\rho|=1$ if and only if $t=0$ and then all points of the front are singular. When $t=0, \zeta_{c}=\zeta_{s}=0$ identically, and the surface degenerates to a single geodesic line. When $t \neq 0$, we have a cylinder with no singularities.

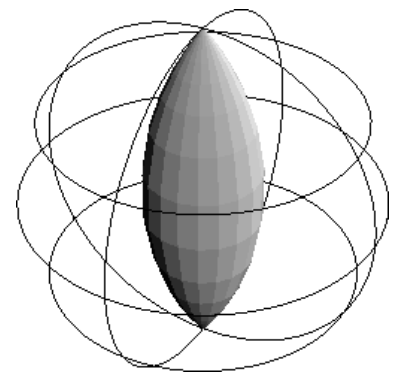


Example 6.2 (Flat fronts of revolution: the snowman and the hourglass). Let $G=z$ and $G_{*}=\mu z$ on $\bar{M}^{2}=\mathbb{C} \cup\{\infty\}$, where $\mu \in \mathbb{R} \backslash\{1\}$. Then $\Delta=e^{-t / 2} z^{1 /(1-\mu)}$. The set of singular points is

$$
\Sigma_{t}=\left\{e^{\sqrt{-1} \beta}\left(\frac{e^{t}|1-\mu|}{\sqrt{|\mu|}}\right)^{\frac{1-\mu}{\mu+1}} ; \beta \in \mathbb{R}\right\} .
$$

Since $\left.\sqrt{\zeta_{c}}\right|_{\Sigma_{t}}= \pm 2 \sqrt{-1}(\mu+1) / \sqrt{\mu}$ is constant, and real if and only if $\mu<0$, the singularities are cuspidal edges when $\mu>0$. When $\mu<0,\left.\zeta_{s}\right|_{\Sigma_{t}}=0$. In this case, the singular points are neither cuspidal edges nor swallowtails, although they are nondegenerate. (The singular image is a single point, but there are many singular points in the domain.)

When $\mu>0$, the image of $\Sigma_{t}$ is a circular cuspidal edge centered about the surface's rotation axis (the snowman, figure on the left). When $\mu<0$, the image of $\Sigma_{t}$ is a single point on the rotation axis (the hourglass, figure on the right).
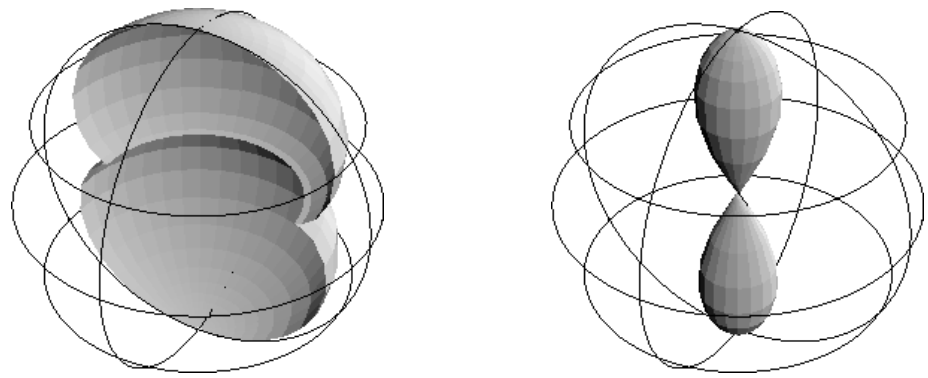

When $\mu=0$, the surface is a horosphere, and when $\mu=-1$, the surface is a hyperbolic cylinder.

When $\mu \rightarrow+1$, the entire surface approaches the ideal boundary $\partial H^{3}$ of $H^{3}$. When $\mu>0$, the corresponding caustic is a cylinder.

Example 6.3 (Peach fronts). Let $G=z+\frac{1}{2}$ and $G_{*}=z-\frac{1}{2}$ on $\bar{M}^{2}=\mathbb{C} \cup\{\infty\}$. Then one has a parallel family of flat fronts $f_{t}: \mathbb{C} \rightarrow H^{3}$ resembling peaches. Since $\Delta=e^{-t / 2} e^{z}$, the set of singular points is $\Sigma_{t}=$ $\{t / 2+\sqrt{-1} y ; y \in \mathbb{R}\}$. Since $\left.\sqrt{\zeta_{c}}\right|_{\Sigma_{t}}= \pm 4 \sqrt{-1}$ is not real, we have a single cuspidal edge along a vertical line on $\mathbb{C}$. This cuspidal edge travels out to the end, hence we have a simple example for which every open neighborhood of the end contains singular points, in particular, each $f_{t}$ is not complete. As noted in [Roitman 2003], the corresponding caustic is the horosphere.

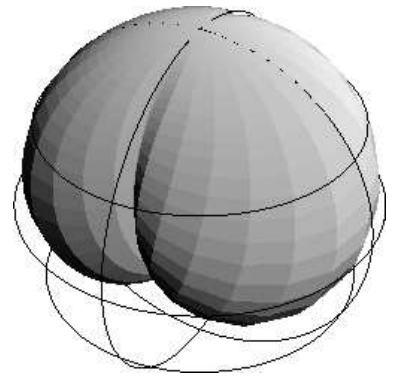


Example 6.4 ( $n$-noid flat fronts). One can make $n$-ended flat fronts for $n \geq 3$ with $G=z, G_{*}=z^{1-n}$ on $M^{2}=\mathbb{C} \cup\{\infty\} \backslash\left\{z ; z^{n}=1\right\}$. For all $t$, the points $z=0, \infty$ are finite and nonsingular. We have $\Delta=e^{-t / 2} \sqrt[n]{z^{n}-1}$,

$$
\begin{aligned}
& \rho(z)=(n-1) e^{-2 t} z^{n-2}\left(z^{n}-1\right)^{(4-2 n) / n}, \\
& \sqrt{\zeta_{c}}= \pm \frac{(n-2)\left(z^{n}+1\right)}{\sqrt{n-1} z^{n / 2}}, \quad \zeta_{s}=\frac{n(2-n)}{2(n-1)} \frac{\left(z^{n}-1\right)^{2}}{z^{n}} .
\end{aligned}
$$

Then $\sqrt{\zeta_{c}}$ is real when $z \in S^{1}$ or $z^{n} \in \mathbb{R}^{+} \backslash\{0\}$. Since

$$
\frac{1}{n-2} \zeta_{c}+\frac{2}{n} \zeta_{s}=\frac{4(n-2)}{n-1}
$$

we get $Z_{c} \cap Z_{s}=\left\{z ; z^{n}=1\right\} \cap M^{2}=\varnothing$; one shows easily that $Z_{0}=\left\{z ; z^{n}=-1\right\}$. In the case $n=3$ (or $n=4$ ), if $6 t>\log 2$ (or $2 t>\log \frac{3}{2}$, respectively), there are twelve (sixteen) swallowtails, and all other singularities are cuspidal edges. If $6 t=\log 2\left(2 t=\log \frac{3}{2}\right)$, there are three (four) degenerate singularities at the points $z^{3}=-1\left(z^{4}=-1\right)$. If $6 t<\log 2\left(2 t<\log \frac{3}{2}\right)$, there are six (eight) swallowtails, and otherwise cuspidal edges. For figures of 3-noids, see [Kokubu et al. 2003]. Here are the caustics corresponding to 3-noids (left) and 4-noids (right):
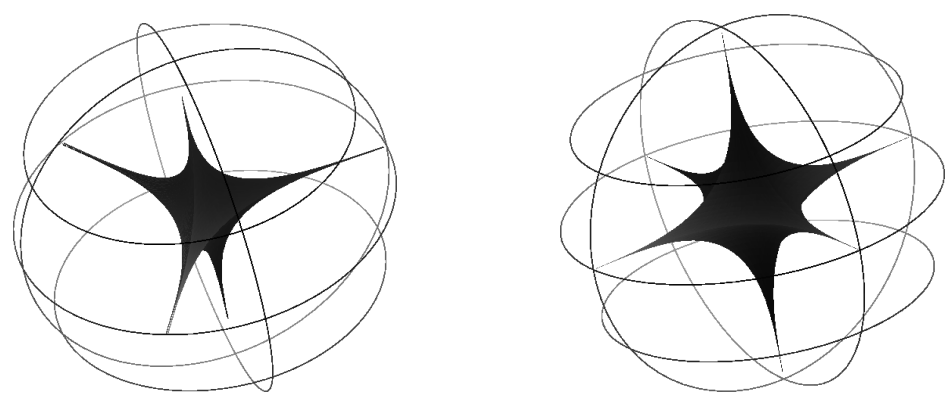

And here are the singular curves in the domain $M^{2}$, for $n=4$ and (from left to right) $2 t>\log \frac{3}{2}, 2 t=\log \frac{3}{2}$ and $2 t<\log \frac{3}{2}$.
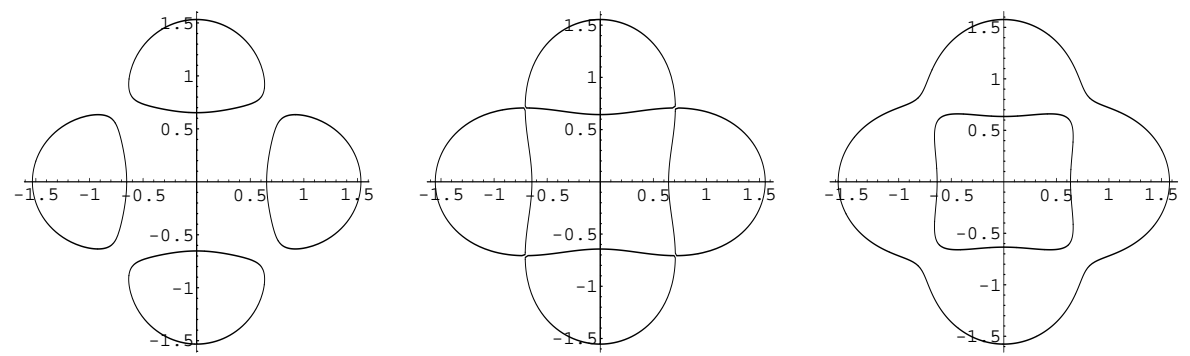
Example 6.5 (Flat fronts with $G=z^{n}$ and $G_{*}=z^{m}$ for $1 \leq n<m$ ). In this case, $M^{2}=\mathbb{C} \backslash\left\{z ; z^{m}=z^{n}\right\}$, and we have $m-n+2$ ends. Then

$$
\begin{aligned}
\Delta & =e^{-t / 2} z^{n}\left(1-z^{m-n}\right)^{n /(n-m)}, \\
\rho(z) & =-\frac{m}{n} e^{-2 t} z^{m+n}\left(1-z^{m-n}\right)^{2(m+n) /(n-m)}, \\
\sqrt{\zeta_{c}} & = \pm \sqrt{-1} \frac{(m+n)\left(z^{m}+z^{n}\right)}{\sqrt{m n}} z^{-(m+n) / 2}, \\
\zeta_{s} & =\frac{m^{2}-n^{2}}{2 m n} z^{-m-n}\left(z^{m}-z^{n}\right)^{2} .
\end{aligned}
$$

For small values of $m$ and $n$, we can easily investigate the singularities.

First consider $n=1$. For $m=2$ (respectively $m=3$ ), for all $t$, all singularities are always cuspidal edges except two (four) swallowtails when $e^{2 t}<\frac{1}{32}\left(\frac{3}{16}\right)$ and at one (two) degenerate singularity (singularities) when $e^{2 t}=\frac{1}{32}\left(\frac{3}{16}\right)$. As the value $e^{2 t}$ increases through $\frac{1}{32}\left(\frac{3}{16}\right)$, the two (four) swallowtails come together into a single (two) degenerate singularity (singularities) and then disappear, leaving only cuspidal edges. The surfaces for $n=1$ and $m=2$ and 3 , and their corresponding caustics, look like this:

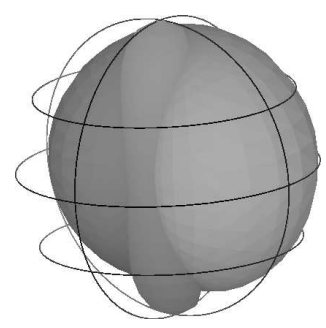

$\left(G, G_{*}\right)=\left(z, z^{2}\right)$

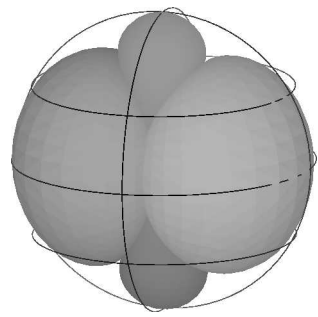

$\left(G, G_{*}\right)=\left(z, z^{3}\right)$

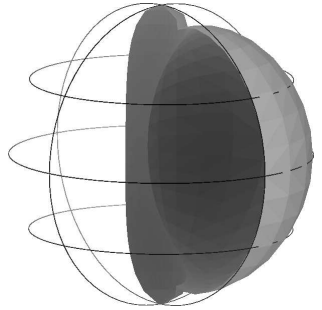

$\left(G, G_{*}\right)=\left(z, z^{2}\right)$ (half cut)

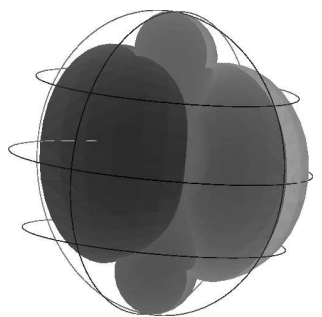

$\left(G, G_{*}\right)=\left(z, z^{3}\right)$ (half cut)

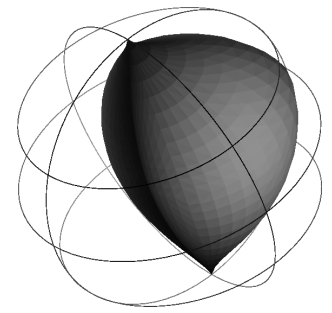

$\left(G, G_{*}\right)=\left(z, z^{2}\right)$ (caustic)

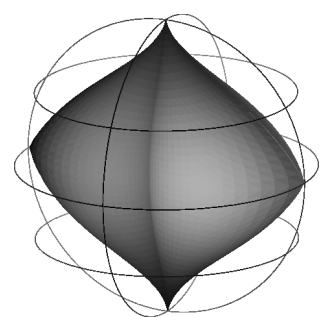

$\left(G, G_{*}\right)=\left(z, z^{3}\right)$ (caustic)

For $n=2$ and $m=3$, and for all $t$, the singular points are always cuspidal edges or swallowtails. All singular points are cuspidal edges, except for one swallowtail when $t<0$. When $t \geq 0$, there are no swallowtails. As $t$ increases to 0 , the 
swallowtail moves out to an end and disappears when $t=0$. For $t=0$, the singular set is the line $\operatorname{Re} z=\frac{1}{2}$, and hence the cuspidal edge travels out to the end $z=\infty$.

And here are the singular curves in the domain $M^{2}$, for $n=1, m=2$ and (from left to right) $e^{2 t}<\frac{1}{32}, e^{2 t}=\frac{1}{32}$ and $e^{2 t}>\frac{1}{32}$.
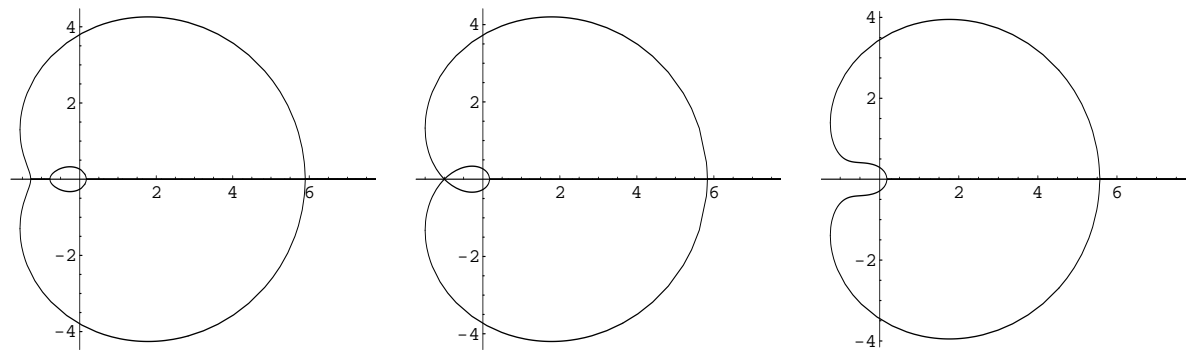

Appendix. Proof of Lemma 2.2

We sketch here the proof of Lemma 2.2 from [Zakalyukin 1983]. We hope this will help readers who are not familiar with singularity theory.

We use three well known facts:

Fact A.1. Let $f: M^{n} \rightarrow \mathbb{R}^{N}$ be an immersion of an $n$-manifold $M^{n}$. Then for each point $p \in M^{n}$, there exists a neighborhood $U$ of $p$ such that the restriction $\left.f\right|_{U}$ is an embedding.

Fact A.2. Let $U_{1}$ and $U_{2}$ be neighborhoods of the origin 0 in $\mathbb{R}^{n}$. Let $f_{i}: U_{i} \rightarrow \mathbb{R}^{N}$ $(i=1,2)$ be two embeddings such that $f_{1}\left(U_{1}\right) \subset f_{2}\left(U_{2}\right)$ and $f_{1}(0)=f_{2}(0)$. Then there exists a local diffeomorphism $\varphi: U_{1} \rightarrow U_{2}$ such that $f_{1}=f_{2} \circ \varphi$ and $\varphi(0)=0$.

It is well-known that a front can be considered as a projection of a Legendrian immersion $L: U \rightarrow P\left(T^{*} \mathbb{R}^{3}\right)$, where $U$ is a domain in $\mathbb{R}^{2}$ and $P\left(T^{*} \mathbb{R}^{3}\right)$ is the projective cotangent bundle. The canonical contact structure of the unit cotangent bundle $T_{1}^{*} \mathbb{R}^{3}$ is the pull-back of that of $P\left(T^{*} \mathbb{R}^{3}\right)$. We remark that this contact structure on $P\left(T^{*} \mathbb{R}^{3}\right)$ does not depend on the Riemannian metric on $\mathbb{R}^{3}$; see [Arnol'd et al. 1985]. Therefore:

Fact A.3. Let $f: U \rightarrow \mathbb{R}^{3}$ be a front, where $U$ is an open subset of $\mathbb{R}^{2}$ and

$$
\Phi: \mathbb{R}^{3} \rightarrow \mathbb{R}^{3}
$$

is a diffeomorphism. Then the composition $\Phi \circ f$ is also a front.

These three facts reduce the theorem to the following proposition:

Proposition A.4. Let $f_{i}: U_{i} \rightarrow \mathbb{R}^{3}(i=1,2)$ be two fronts satisfying $f_{1}(0)=f_{2}(0)$, whose associated Legendrian immersions $L_{f_{i}}: U_{i} \rightarrow T_{1}^{*} \mathbb{R}^{3}$ are embeddings, where $U_{i}$ are neighborhoods of the origin 0 in $\mathbb{R}^{2}$. Suppose that there exists a relatively compact neighborhood $V_{i}$ of $0(i=1,2)$ such that 
(1) The closure $\bar{V}_{i}$ is contained in $U_{i}$ for $i=1,2$.

(2) The set of regular points of $f_{i}$ in $\bar{V}_{i}$ is dense in $\bar{V}_{i}(i=1,2)$.

(3) $f_{1}\left(\bar{V}_{1}\right)=f_{2}\left(\bar{V}_{2}\right)$

Then $L_{f_{1}}\left(\bar{V}_{1}\right)=L_{f_{2}}\left(\bar{V}_{2}\right)$.

Before proving this proposition, we give the proof of Lemma 2.2:

Proof of Lemma 2.2. Statement (i) of the lemma follows from (ii) immediately. So it is sufficient to show (i) implies (ii). By Fact A.3, we may assume $f_{1}\left(V_{1}\right)=$ $f_{2}\left(V_{2}\right)$. Without loss of generality we may assume that $V_{1}$ and $V_{2}$ are relatively compact and $\bar{V}_{1}, \bar{V}_{2} \subset U$. By Fact A.1, we may assume that the associated Legendrian immersion $L_{f_{i}}: U \rightarrow T_{1}^{*} \mathbb{R}^{3}$ is an embedding. Since $V_{1}$ and $V_{2}$ are relatively compact, we have

$$
f_{1}\left(\bar{V}_{1}\right)=\overline{f_{1}\left(V_{1}\right)}=\overline{f_{2}\left(V_{2}\right)}=f_{2}\left(\bar{V}_{2}\right) .
$$

Thus by Proposition A.4, we have $L_{f_{1}}\left(\bar{V}_{1}\right)=L_{f_{2}}\left(\bar{V}_{2}\right)$, in particular we have

$$
L_{f_{1}}\left(V_{1}\right) \subset L_{f_{2}}(U) \text {. }
$$

By Fact A.2, there exists a local diffeomorphism $\varphi$ on $\mathbb{R}^{2}$ such that $L_{f_{2}}=L_{f_{1}} \circ \varphi$, which proves the assertion.

To prove the Proposition A.4, we set

$$
\begin{aligned}
S & =f_{1}\left(\bar{V}_{1}\right)=f_{2}\left(\bar{V}_{2}\right), \\
Z_{i} & =\left\{f_{i}(p) \in S ; p \in \bar{V}_{i} \text { is a singular point of } f_{i}\right\} \quad(i=1,2), \\
Z & =Z_{1} \cup Z_{2}, \quad R=S \backslash Z,
\end{aligned}
$$

and first prove the following simple lemma:

Lemma A.5. For each $a \in S \backslash Z_{i}, f_{i}^{-1}(a)$ is a finite set.

Proof. Suppose that $f^{-1}(a)$ is not a finite set. Without loss of generality, we can take a sequence $\left\{p_{n}\right\}$ such that

$$
f_{i}\left(p_{n}\right)=a \quad(n=1,2, \ldots) .
$$

Moreover, by taking a subsequence we may assume $\left\{p_{n}\right\}$ converges to a point $p \in \bar{V}_{i}$. Then by continuity, we have $f_{i}(p)=a$. Since $a \in S \backslash Z_{i}, p$ is a regular point of $f_{i}$. Thus, there exists a neighborhood $V$ of $p$ such that $\left.f_{i}\right|_{V}$ is an embedding, which contradicts

$$
f_{i}\left(p_{n}\right)=a=f_{i}(p),
$$

since $p_{n} \in V$ for sufficiently large $n$. 
Proof of Proposition A.4. Fix $a \in R$ arbitrarily. By the previous lemma, we can set

$$
f_{1}^{-1}(a)=\left\{p_{1}, \ldots, p_{m}\right\}, \quad f_{2}^{-1}(a)=\left\{q_{1}, \ldots, q_{l}\right\} .
$$

We identify $T_{1}^{*} \mathbb{R}^{3}$ with $T_{1} \mathbb{R}^{3}=\mathbb{R}^{3} \times S^{2}$. Then $L_{f_{i}}(i=1,2)$ is considered as a map into $\mathbb{R}^{3} \times S^{2}$, and there exist unit vectors $v_{1}, \ldots, v_{m}$ and $\xi_{1}, \ldots, \xi_{\ell}$ such that

$$
L_{f_{1}}\left(p_{j}\right)=\left(a, v_{j}\right), \quad L_{f_{2}}\left(q_{k}\right)=\left(a, \xi_{k}\right) \quad(j=1, \ldots, m, k=1, \ldots, l) .
$$

Since $L_{f_{1}}$ and $L_{f_{2}}$ are embeddings, $v_{1}, \ldots, v_{m}$ (resp. $\xi_{1}, \ldots, \xi_{l}$ ) are mutually distinct. Thus the image of $f_{i}$ at $a$ consists of a mutually transversal finite number of components of surfaces. Since $f_{1}\left(\bar{V}_{1}\right)=f_{2}\left(\bar{V}_{2}\right)$, we can conclude that $m=l$ and

$$
L_{f_{1}}\left(p_{j}\right)=\left(a, v_{j}\right)=\left(a, \xi_{j}\right)=L_{f_{2}}\left(q_{j}\right) \quad(j=1,2, \ldots, m)
$$

for a suitable permutation of $p_{1}, \ldots, p_{m}$. Now we set

$$
W_{1}=\left(\left.f_{1}\right|_{\bar{V}_{1}}\right)^{-1}(R), \quad W_{2}=\left(\left.f_{2}\right|_{\bar{V}_{2}}\right)^{-1}(R) .
$$

By $(\mathrm{A}-1)$, we have

$$
L_{f_{1}}\left(W_{1}\right)=L_{f_{2}}\left(W_{2}\right) .
$$

Then by the continuity of $L_{f_{1}}$ and $L_{f_{2}}$, we have

$$
L_{f_{1}}\left(\bar{W}_{1}\right)=L_{f_{2}}\left(\bar{W}_{2}\right) .
$$

Thus it is sufficient to show that $W_{i}$ is dense in $\bar{V}_{i}$. Suppose that $\left(\left.f_{i}\right|_{\bar{V}_{i}}\right)^{-1}(Z)$ has an interior point. By assumption (2) of Proposition A.4, there exists an open subset $O_{i} \subset V_{i}$ such that $f_{i}\left(O_{i}\right) \subset Z$ and $f_{i}$ is an immersion on $O_{i}$. Take a point $q_{i} \in O_{i}$. Let $T_{i}$ be the tangent plane (as a two dimensional affine plane in $\mathbb{R}^{3}$ ) of the regular surface $f_{i}\left(O_{i}\right)$ at $q_{i}$, and

$$
\pi_{i}: \mathbb{R}^{3} \rightarrow T_{i} \quad(i=1,2)
$$

the orthogonal projection. Since $\pi_{i} \circ f_{i}$ has a regular point, $\pi_{i} \circ f_{i}\left(O_{i}\right)$ contains an interior point. On the other hand, by Sard's theorem, the critical value set $\pi_{i}(Z)$ of $\pi_{i} \circ f_{i}$ is a measure zero set. Since $\pi_{i} \circ f_{i}\left(O_{i}\right) \subset \pi_{i}(Z)$, this makes a contradiction. Hence $\left(\left.f_{i}\right|_{\bar{V}_{i}}\right)^{-1}(Z)$ does not have any interior points. Since

$$
\left(\left.f_{i}\right|_{\bar{V}_{i}}\right)^{-1}\left(\bar{V}_{1}\right)=\left(\left.f_{i}\right|_{\bar{V}_{i}}\right)^{-1}(R \cup Z)=\left(\left.f_{i}\right|_{\bar{V}_{i}}\right)^{-1}(R) \cup\left(\left.f_{i}\right|_{\bar{V}_{i}}\right)^{-1}(Z),
$$

$W_{i}=\left(\left.f_{i}\right|_{\bar{V}_{i}}\right)^{-1}(R)$ is dense in $\bar{V}_{i}$.

The authors' original proof of Proposition A.4 used the Hausdorff dimension of $f_{i}\left(O_{i}\right)$. Go-o Ishikawa pointed out to us a simplification of the proof that requires only the classical Sard's theorem. 


\section{Acknowledgements}

The authors thank Shyuichi Izumiya and Go-o Ishikawa for fruitful discussions and valuable comments. Ishikawa kindly pointed out an error in a preliminary version of our paper.

\section{References}

[Arnol'd et al. 1985] V. I. Arnol'd, S. M. Guseı̌n-Zade, and A. N. Varchenko, Singularities of differentiable maps, vol. 1, Monographs in Mathematics 82, Birkhäuser, Boston, 1985. MR 86f:58018 Zbl 0554.58001

[Bruce and Giblin 1984] J. W. Bruce and P. J. Giblin, Curves and singularities: a geometrical introduction to singularity theory, Cambridge University Press, Cambridge, 1984. MR 87m:58021 Zbl 0534.58008

[Fujimori et al. 2005] S. Fujimori, K. Saji, M. Umehara, and K. Yamada, "Cuspidal cross caps and singularities of maximal surfaces", preprint, 2005.

[Gálvez et al. 2000] J. A. Gálvez, A. Martínez, and F. Milán, "Flat surfaces in the hyperbolic 3space”, Math. Ann. 316:3 (2000), 419-435. MR 2002b:53013 Zbl 1003.53047

[Golubitsky and Guillemin 1973] M. Golubitsky and V. Guillemin, Stable mappings and their singularities, Graduate Texts in Math. 14, Springer, New York, 1973. MR 49 \#6269 Zbl 0294.58004

[Izumiya and Takeuchi 2003] S. Izumiya and N. Takeuchi, "Geometry of ruled surfaces", pp. 305338 in Applicable mathematics in the golden age, edited by J. C. Misra, Narosa, New Delhi, 2003. [Kitagawa 1988] Y. Kitagawa, "Periodicity of the asymptotic curves on flat tori in $S^{3}$ ", J. Math. Soc. Japan 40:3 (1988), 457-476. MR 89g:53080 Zbl 0642.53059

[Kitagawa 1995] Y. Kitagawa, "Embedded flat tori in the unit 3-sphere", J. Math. Soc. Japan 47:2 (1995), 275-296. MR 96e:53093 Zbl 0836.53035

[Kitagawa 2000] Y. Kitagawa, "Isometric deformations of flat tori in the 3-sphere with nonconstant mean curvature", Tohoku Math. J. (2) 52:2 (2000), 283-298. MR 2001f:53130 Zbl 0995.53046

[Kobayashi and Nomizu 1963] S. Kobayashi and K. Nomizu, Foundations of differential geometry, vol. I, Wiley-Interscience, New York, 1963. MR 27 \#2945 Zbl 0119.37502

[Kokubu et al. 2003] M. Kokubu, M. Umehara, and K. Yamada, "An elementary proof of Small's formula for null curves in PSL $(2, \mathbb{C})$ and an analogue for Legendrian curves in PSL(2, $\mathbb{C})$ ", Osaka J. Math. 40:3 (2003), 697-715. MR 2004g:53011 Zbl 1042.53042

[Kokubu et al. 2004] M. Kokubu, M. Umehara, and K. Yamada, "Flat fronts in hyperbolic 3-space", Pacific J. Math. 216:1 (2004), 149-175. MR 2005f:53021 Zbl 02138440

[Kokubu et al. 2005] M. Kokubu, W. Rossman, M. Umehara, and K. Yamada, "Flat fronts in hyperbolic 3-space and their caustics", preprint, 2005.

[Langevin et al. 1995] R. Langevin, G. Levitt, and H. Rosenberg, "Classes d'homotopie de sur-

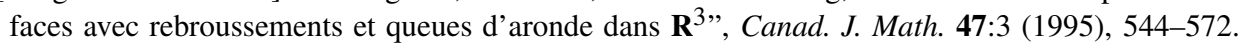
MR 96j:57040 Zbl 0837.57025

[Roitman 2003] P. Roitman, "Flat surfaces in hyperbolic 3-space as normal surfaces to a congruence of geodesics", preprint, Universidade de Brasília, 2003.

[Zakalyukin 1983] V. M. Zakalyukin, "Reconstructions of fronts and caustics depending on a parameter and versality of mappings", pp. 56-93 in Sovremennye problemy matematiki, vol. 22, edited by R. V. Gamkrelidze, Akad. Nauk SSSR Vsesoyuz. Inst. Nauchn. i Tekhn. Inform., Moscow, 1983. In Russian; translation in J. Soviet Math. 27 (1984), 2713-2735. MR 85h:58029 Zbl 0554.58011 
Received December 27, 2003. Revised December 10, 2004.

\author{
Masatoshi KOKUBU \\ DEPARTMENT OF NATURAL SCIENCE \\ SCHOOL OF ENGINEERING \\ TOKYO DENKI UNIVERSITY \\ 2-2 KANDA-NISHIKI-CHO \\ CHIYODA-KU, TOKYO 101-8457 \\ JAPAN \\ kokubu@cck.dendai.ac.jp \\ WAYNE ROSSMAN \\ DEPARTMENT OF MATHEMATICS \\ FACULTY OF SCIENCE \\ KOBE UNIVERSITY \\ ROKKO, КоBE 657-8501 \\ JAPAN \\ wayne@math.kobe-u.ac.jp \\ KENTARO SAJI \\ DEPARTMENT OF MATHEMATICS \\ HOKKAIDO UNIVERSITY \\ SAPPORO 060-0810 \\ JAPAN \\ saji@math.sci.hokudai.ac.jp \\ MASAAKI UMEHARA \\ DEPARTMENT OF MATHEMATICS \\ GRAdUATE SCHOOL OF SCIENCE \\ OSAKA UNIVERSITY \\ TOYONAKA, OSAKA 560-0043 \\ JAPAN \\ umehara@math.wani.osaka-u.ac.jp \\ KOTARO YAMADA \\ FACULTY OF MATHEMATICS \\ KYUSHU UNIVERSITY \\ HIGASHI-KU \\ FUKUOKA 812-8581 \\ JAPAN \\ kotaro@math.kyushu-u.ac.jp
}

